G.C. Gebel, T. Mosbach, W. Meier, M. Aigner, Optical and spectroscopic diagnostics of laser-induced air breakdown and kerosene spray ignition, Combust. Flame 162 (2015) 1599-1613.

The original publication is available at www.elsevier.com

http://dx.doi.org/10.1016/j.combustflame.2014.11.024 


\title{
Optical and spectroscopic diagnostics of laser-induced air breakdown and kerosene spray ignition
}

\author{
Gregor C. Gebel*, Thomas Mosbach, Wolfgang Meier, and Manfred Aigner
}

Institute of Combustion Technology, German Aerospace Center (DLR), 70569 Stuttgart, Germany

\begin{abstract}
This study focuses on the transition from a laser-induced breakdown plasma to a flame kernel in two-phase flows. The test rig was a vertical flow channel with a full-cone spray nozzle installed inside. The fuel was Jet A1 aviation kerosene. Breakdowns were generated by the focused laser pulses from a frequency-doubled and Qswitched Nd:YAG laser. The investigation of laser-induced breakdowns in ambient air provided valuable supplementary data to understand the interaction of the breakdown plasma and the fuel spray. To determine the breakdown energy, the amount of absorbed laser pulse energy was measured, and the blast wave energy consumption was estimated. Blast waves were visualized with high-speed schlieren imaging. Their energies were estimated by the application of Jones' blast wave expansion model. High-speed imaging of air and spray breakdowns visualized their transient morphologies. Expansion velocities of air breakdowns were determined and revealed a supersonic expansion during the first few microseconds. Air breakdowns decayed and disappeared within $30 \mu \mathrm{s}$. Spray beakdowns were observed over a period of $90 \mu \mathrm{s}$, which covered their transition into flame kernels. Optical emission spectroscopy was applied to ambient air breakdowns, spray ignitions and spray breakdowns in nitrogen. The temporal decrease of nitrogen ion and atom lines was investigated, and mean lifetimes were determined. $\mathrm{CN}^{*}, \mathrm{C}_{2}{ }^{*}$ and $\mathrm{CH}^{*}$ radicals were observed in spray ignitions, but no $\mathrm{CH}^{*}$ was confirmed in spray breakdowns in nitrogen, while $\mathrm{CN}^{*}$ and $\mathrm{C}_{2} *$ occurred with a similar intensity as in spray ignitions. Simulated spectra were fitted to the $\mathrm{CN}^{*} \mathrm{~B}^{2} \Sigma^{+}-\mathrm{X}^{2} \Sigma^{+}$band between 384.2 and $388.4 \mathrm{~nm}$ to determine temperatures at the breakdown region during the transition from plasma into spray flame kernels.
\end{abstract}

Keywords: Ignition, kerosene, spray, laser-induced breakdown, flow visualization, spectroscopy

* Corresponding author.

E-mail: gregor.gebel@dlr.de

Telephone: +497116862437

Fax: $\quad+497116862537$

\section{Introduction}

The specific background of this study is the relight of aviation gas turbines at high altitude. Their ability to ignite at the low air densities and temperatures at high flight levels is a key certification requirement [1]. Modern low- $\mathrm{NO}_{\mathrm{x}}$ combustors run on lean mixtures, which increase the challenge of high altitude relight $[2,3]$. The 
development of such combustors can benefit from numerical simulations of the ignition process, because an iterative development including extensive testing is time-consuming and cost-intensive. In the first stage of an ignition, a spark turns into a small self-sustaining combustion, a 'flame kernel'. The involved physical and chemical processes are quite complex and not yet well understood. With the present knowledge, the simulation of a flame kernel generation for a two-phase flow ignition is only possible if the real physics are strongly simplified. Therefore, a better understanding of the involved mechanisms is necessary to develop more accurate models. Experiments in laboratory test rigs with well-defined boundary conditions provide very important contributions. Ignition by electrical spark discharge is the most common method in technical applications. But a spark plug has some disadvantages in a laboratory experimental setup: Shifting of the ignition location is restricted. Boundary conditions are complicated, because the plug is a heat sink and interacts with the flow field. Variation of the ignition energy is restricted and of low repeatability, and the triggering accuracy is low. Therefore, laserinduced ignition is a good alternative. Four different laser ignition mechanisms are known [4]:

- Thermal initiation by the absorption of laser radiation through rovibrational molecule bands.

- Non-resonant breakdown, initiated by multiphoton ionization and followed by electron cascade breakdown.

- Resonant breakdown, initiated by photodissociation and followed by electron cascade breakdown.

- Photochemical ignition through radical production by photolysis.

The mechanism with the greatest physical similarity to electrical spark discharge is the non-resonant breakdown, although its electric field strength is higher by several orders of magnitude. We applied this mechanism with a Q-switched and frequency-doubled Nd:YAG laser. It offered the following advantages:

1. No spark plug with the above-named restrictions was required.

2. The triggering accuracy was $\pm 1 \mathrm{~ns}$, supporting precise synchronization with diagnostic instruments.

3. A flexible selection of breakdown location and time.

4. Continuous laser pulse energy adjustment between 30 and $300 \mathrm{~mJ}$.

5. Very good reproducibility.

The first report of laser-induced breakdown was given in 1963 by Maker et al. [5]. Its first usage for the ignition of a gas mixture was reported by Lee and Knystautas in 1969 [6]. Since then, comprehensive research investigated both, laser-induced breakdown in inert gases and laser-induced ignition of gas mixtures. A review of significant results is provided by Phuoc [7]. Considerably less research investigated the laser-induced ignition of two-phase flows. In 1998, Oldenborg et al. [8] reported on the investigation of laser-induced spray ignition for aviation gas turbines. Their intention was to develop a concept for laser-based igniters, which can increase reliability and reduce $\mathrm{NO}_{\mathrm{x}}$ emissions. They ignited Jet A sprays using an Nd:YAG and a Cr:LiSAF laser. Various configurations were tested, including ultra-cold conditions, single and multiple laser pulses. They found a high potential of laser-induced spray ignition and developed some concepts for technical application, but those were 
never introduced to aviation gas turbines. A major disadvantage was the large size of the laser systems available at that time.

In recent years, the interest in laser-based spray ignition systems has substantially grown for two reasons. First, laser technology has progressed significantly. Compact diode-pumped solid-state (DPSS) lasers feature a high potential for application in engines [9,10]. High-power fibers are available to guide the radiation from the laser to the combustor [11]. Second, present demands on technical combustion systems, such as better reliability, safety, efficiency, lower emissions and fuel flexibility require advanced combustion concepts. This also affects the ignition systems. Therefore, laser-induced spray ignition is considered for reciprocating engines [1215], aviation gas turbines [16-18], and spacecraft thrusters [19]. In particular, reciprocating engines and aviation gas turbines benefit from the possibility to adjust the ignition position. No spark plugs close to the combustor walls are necessary, which reduces the risk of flame kernel quenching on cold metal parts. Also, ignition at multiple and variable positions is possible. This opens new options for combustor geometries and contributes significantly to increased efficiency and reduced emissions. Moreover, laser-based igniters can be the key to reliable ignition of lean mixtures, and a high potential is seen for the application in lean premixed prevaporized (LPP) combustion [17,18].

In conclusion, the demand for the present study arises from two aspects. First, insights into fundamental processes of two-phase flow ignition are required for technical applications and for the development of advanced numerical models. Second, laser-induced ignition is a state-of-the-art technology with a high potential for the application in future combustion engines. The following questions have to be answered to make effective use of this technology:

- How do fuel droplets affect the formation and subsequent development of the breakdown?

- How does the breakdown develop into a flame kernel (time scales, spatial development, conditions inside the breakdown)?

- What is the impact of the breakdown on fuel droplets in the immediate and wider vicinity of the breakdown site (heat transfer, blast wave)?

- What are the consequences of this impact for the subsequent flame kernel growth?

These questions give raise to our research. The study presented in this paper supplements our previous publications [20-22]. While they focus on the gas dynamics around the breakdown (particularly the blast wave) and their breakup of fuel droplets, this study focuses on the transition of a laser-induced fuel spray breakdown into a flame kernel. The following investigations were performed:

- The amount of laser pulse energy absorbed by air breakdowns was determined with a volume absorber.

- High-speed schlieren imaging was used to track the expansion trajectory of laser-induced blast waves in ambient air, in order to estimate their energy consumption.

- High-speed imaging was used to visualize the transient morphology of laser-induced air breakdowns and spray ignitions. 
- Optical emission spectroscopy was used to determine the physical nature of the breakdowns, including species and temperatures.

The investigation of laser-induced air breakdowns provided valuable supplementary data to understand the interaction of the fuel spray and the breakdown plasma. The fuel was Jet A-1 aviation kerosene. The spray was provided by an air-assisted siphon nozzle.

Literature on laser induced two-phase flow ignition is very limited. But some aspects with particular relevance to the present study were addressed in previous publications, and a short review is provided in the subsequent paragraphs.

Lawes et al. [12] investigated the laser-induced ignition of monodisperse iso-octane aerosols. The aerosols were generated in a Wilson cloud chamber, and the ignition laser was a Q-switched Nd:YAG laser at $1064 \mathrm{~nm}$. Multiple breakdowns occurred along the laser beam path through the cloud at a high pulse energy of $270 \mathrm{~mJ}$. Each breakdown was the origin of a spherical blast wave. A low pulse energy of $32 \mathrm{~mJ}$ resulted in a single breakdown at the focal point. A very similar observation was made by Kawahara et al. [15]. They ignited ethanol sprays with a Q-switched Nd:YAG laser at $532 \mathrm{~nm}$. They found multiple breakdown generation along the laser beam path. Each breakdown was the origin of a blast wave, which caused droplet dispersion. Mösl et al. [18] investigated the potential of laser-ignition systems for aviation gas turbines with a Jet A-1 kerosene spray. A Q-switched Nd:YAG laser at $1064 \mathrm{~nm}$ and $100 \mathrm{~mJ}$ pulse energy was compared to a spark plug of $1.8 \mathrm{~J}$ spark energy. The ignition probabilities at different breakdown locations were determined. High ignition probabilities for both methods were obtained at the spray cone edge and in the recirculation zone. But due to the significantly higher energies, electrical spark discharges revealed slightly lower lean ignition limits than the laser-induced breakdowns. The cited investigations show that, laser-induced breakdowns should be located near the spray cone edge on the laser beam incident side. Energy losses by laser pulse scattering on droplets are avoided, and the good mixing at the spray cone edge supports the ignition process.

The experiments of El-Rabii et al. [16] gave indication of the minimum laser pulse energies required to ignite fuel sprays. They measured minimum ignition energies (MIE) in n-heptane and JP-4 (military jet fuel) droplet clouds. The clouds were created by an ultrasonic atomizer, which generated a mean droplet diameter of $31 \mu \mathrm{m}$. Breakdowns were induced by a Q-switched Nd:YAG laser at $1064 \mathrm{~nm}$. The MIE was found to be between 16 and $30 \mathrm{~mJ}$ laser-pulse energy for different fuel-air ratios. MIE depends on various aspects related to the experimental setup, the quality of mixture formation, the flow conditions and gas dynamics at the ignition location. The study demonstrated that, ignition is basically possible at relatively low pulse energies. In contrast, electrical spark igniters have energy inputs of 1 to $12 \mathrm{~J}$ [23]. Our main interest was the investigation of fundamental aspects at conditions similar to technical applications. Therefore, we applied a relatively high laser pulse energy near $200 \mathrm{~mJ}$.

An experimental investigation of the laser-induced ignition of methane and propane-air mixtures by Beduneau et al. [24] showed that, the success of an ignition depends less on the flame kernel size but more on the radical generation during the first few microseconds. This might only be true for ideally premixed gases, and things are very different in sprays, as demonstrated by Letty et al. [25]. They investigated the ignition probabil- 
ity of an n-heptane spray in a bluff body burner. A Q-switched Nd:YAG laser at $1064 \mathrm{~nm}$ provided $140 \mathrm{~mJ}$ pulses. A significant contribution of the study was the definition of three different ignition failure modes, which were characterized by the flame kernel lifetime: short (few micro- to milliseconds), intermediate (few tens of milliseconds) and long (hundreds of milliseconds). The modes illustrate that, spray ignition may still fail after a flame kernel was successfully generated. In the present study, the abundant laser pulse energy of nearly $200 \mathrm{~mJ}$ always created flame kernels, which developed into stable spray flames with a probability of $100 \%$.

\section{Experimental setup}

\subsection{Flow channel}

The experiments were performed in a vertical flow channel with a square cross-section of $62 \mathrm{~cm}^{2}$ and a center section length of one meter. Three sides were equipped with windows to provide access for optical measurement techniques. The fourth side was mounted to a transverse system, which allowed horizontal and vertical shifting. A schematic of the flow channel is shown in Figure 1. A continuous, top-down air co-flow could be established to supply the combustion zone with air and to flush out fuel vapour and fumes. The co-flow inlet was a sinter metal ring on top of the center section. The air co-flow was $862 \mathrm{~g} / \mathrm{min}$. The spray nozzle was a Delavan SN 30609-2 air-assisted siphon nozzle. It was installed inside the flow channel and injected the fuel downstream into the air co-flow. The fuel was directed to the nozzle though a vertical pipe inside the flow channel. A second pipe supplied the nozzle with an atomization air flow, which circumferentially impacted the fuel jet just before the nozzle exit. The primary breakup zone reached about $5 \mathrm{~mm}$ downstream. The spray was a slightly swirling full-cone spray with a cone angle of approximately 40 degrees at the nozzle exit and a droplet diameter distribution peaking between 20 and $50 \mu \mathrm{m}$. The spray was investigated in more detail in a previous study [26]. The fuel was a commercial Jet A-1 aviation kerosene from the international airport of Stuttgart, Germany. Its temperature was controlled to be $294 \mathrm{~K}$ with an accuracy of $\pm 0.2 \mathrm{~K}$. The fuel mass flow was $20 \mathrm{~g} / \mathrm{min}$, and the atomization air mass flow was $7.5 \mathrm{~g} / \mathrm{min}$.

\subsection{Ignition laser}

The ignition laser was a frequency-doubled and Q-switched Nd:YAG laser model InnoLas Spitlight 600-10. It provided nearly Gaussian (spatial and temporal) pulses of up to $300 \mathrm{~mJ}$ at $532 \mathrm{~nm}$, a pulse length of $6 \mathrm{~ns}$ and a trigger accuracy of $\pm 1 \mathrm{~ns}$ in a single pulse mode. The beam path is illustrated in Figure 2 . The beam was steered to an optical bench with a periscope, where it was expanded to a diameter of approximately $40 \mathrm{~mm}$ with a Galilean telescope. The expanded beam was directed into the flow channel with a downward angle of 23 degrees and refocused directly in front of the inlet window by a laser-grade doublet lens with a focal length of $120 \mathrm{~mm}$. The angle of the converging laser beam was determined to be approximately 10 degrees. The expansion of the laser beam served two interests: First, the doublet lens produced a tight focus. Second, the irradiance at the inlet window was reduced to avoid thermal stress and surface damage. The focal point was positioned close to the 
spray cone edge $20 \mathrm{~mm}$ below the nozzle and $4 \mathrm{~mm}$ off the spray center axis. The ignition reliability was $100 \%$ due to the high level of the laser pulse energy near, see section 3.

\subsection{Schlieren imaging}

Schlieren images were recorded with a high-speed CMOS camera model Photron / LaVision HighSpeedStar 6. The camera viewed the breakdown region though the same window which gave access for the laser pulse. A representation of the experimental set-up is shown in Figure 2. Please note, the figure illustrates the schlieren imaging, high-speed imaging, and optical emission spectroscopy setup. The setups were not applied simultaneously. The light source was a halogen projector lamp. Its light beam was collimated by a 4" lens ( $\mathrm{f}=$ $600 \mathrm{~mm}$ ) to a diameter of approximately $90 \mathrm{~mm}$. The beam passed the flow channel and was refocused by a similar lens on the opposite side. The camera was equipped with a Tokina $100 \mathrm{~mm} \mathrm{f} / 2.8$ macro lens. It was operated at a sustained repetition rate of $50 \mathrm{kHz}$, an exposure time of $1 \mu \mathrm{s}$ and a resolution of $320 \times 288$ pixels. The field-of-view was determined to be $46 \times 42 \mathrm{~mm}$ with a calibration target. The camera and the laser were externally triggered by a combination of a BNC 565 and a Quantum 9520 trigger generator. The Quantum 9520 gave the continuous trigger signals for the camera controller and for the $10 \mathrm{~Hz}$ laser flash lamp. The BNC 565 was synchronized with the Quantum 9520, and was started by the test rig control computer. It gave the trigger signals for the laser Q-switch and the camera exposure start. The Schlieren effect was generated with an iris aperture, which was adjusted to cut with one lamella into the focal point from one side, in order to visualize vertical density gradients.

\subsection{High-speed imaging}

The HighSpeedStar 6 camera was arranged perpendicular to the laser beam path and observed the breakdown from a sideways perspective through a quartz window. The position of the high-speed camera relative to the flow channel is shown in Figure 2. The camera has a spectral sensitivity range from 350 to $1000 \mathrm{~nm}$. The quantum efficiency of the image sensor has its maximum in the spectral range between 410 and $630 \mathrm{~nm}$. The camera was equipped with a Tokina $100 \mathrm{~mm} \mathrm{f} / 2.8$ macro lens. It provided broadband luminosity images at a sustained repetition rate of $250 \mathrm{kHz}$, an exposure time of $1 \mu \mathrm{s}$ and a resolution of $128 \times 80$ pixels. The field of view was determined to be $10.7 \times 6.69 \mathrm{~mm}$ with a calibration target. The external trigger system was similar to that used for the schlieren imaging.

\subsection{Optical emission spectroscopy}

Breakdown spectra were recorded for air and spray breakdowns. The spectrometer was an LLA Instruments ESA $4000 \mathrm{EV} / \mathrm{i}$ échelle spectrometer with a spectral range from 200 to $870 \mathrm{~nm}$. The emissions from ambient air breakdowns were captured with an optical setup consisting of a Glan-Thompson polarizer, a lens and a small quartz collimator. The optics were transparent for the near infrared, visible, and near ultraviolet spectrum. The polarizer reduced scatter light and reflections of the laser pulse, and the lens focused the light on the smaller 
collimator. The optics were placed perpendicular to the laser beam path and as close to the breakdown as possible. The distance between the focal point and the optics was $34 \mathrm{~mm}$. The emissions from spray breakdowns were captured with a 1" collimator which was nontransparent for ultraviolet light. Consequently, the spectra show a steep intensity decline below $370 \mathrm{~nm}$. The collimator was mounted outside the flow channel and viewed the breakdown through a quartz window. The distance between the focal point and the lens was approximately $50 \mathrm{~mm}$. Breakdown emissions were directed from the collimation optics to the spectrometer with an optical fiber. The spectrometer was calibrated for both optical setups with a tungsten-deuterium lamp. The laser flash lamp pulsed with $10 \mathrm{~Hz}$ on an internal clock. The laser Q-switch and the spectrometer were externally triggered by a BNC 565 trigger generator. A representation of the experimental set-up is shown in Figure 2, showing the approximate position of the collimation optics and the fiber connection to the spectrometer.

\section{Breakdown and blast wave energy in air}

We created laser-induced breakdowns by the non-resonant multiphoton and electron cascade process, which is described in more detail by Phuoc [7]. Initial temperatures reach an order of magnitude of up to $10^{6} \mathrm{~K}$ while the laser pulse is still in progress [27]. They decay rapidly to an order of magnitude of $10^{4} \mathrm{~K}$ after the pulse has terminated [28]. A fundamental information is the energy contained in the breakdown. The laser pulse energy is not completely absorbed. Losses may include transmission, scattering and diffraction. Furthermore, while the breakdown plasma expands, energy is transferred into a laser-supported detonation wave (LSD wave), which is the shock layer at the interface between the plasma and the surrounding area [29]. When the expansion decelerates, the wave detaches and expands as a nearly spherical blast wave. The amount of absorbed pulse energy in the breakdown region was determined by volume absorber measurements, which is presented in section 3.1. The blast wave energy was determined by the analysis of schlieren images, which is presented in section 3.2. The blast wave energy is not completely lost within a spray ignition, but it contributes to the secondary breakup of fuel droplets in the vicinity of the breakdown. This effect is discussed by the authors in two other publications [20,21]. The remaining breakdown energy is consumed by different thermal energy transfer mechanisms, in particular by radiation, conduction, convection, evaporation, plasma and chemical processes. These mechanisms create favorable conditions for the initiation of combustion reactions. Together, the measurements in section 3.1 and 3.2 determined the air breakdown energy. The result is provided at the end of section 3.2. Equivalent measurements for the spray ignition were not possible due to the disturbances of liquid fuel on both, volume absorber measurements and schlieren imaging. The breakdown and blast wave energy in fuel spray is expected to be be somewhat smaller than the air breakdown energies, since a fraction of the laser pulse energy is lost by Mie scattering on fuel droplets.

\subsection{Breakdown energy}

The laser pulse energy directly in front of and behind the breakdown position was measured with a laser power volume absorber type Gentec-EO UP19K-15S-VR. The measurement was exclusively done for air breakdowns, because Mie scattering of the laser pulse on fuel droplets would prevent any measurement. The volume absorber 
was placed about $20 \mathrm{~mm}$ in front of the focal point. Photons have already passed all optical components of the ignition system at this position, but the irradiance is too low for breakdown initiation. In a separate measurement, the volume absorber was placed about $20 \mathrm{~mm}$ behind the focal point, in order to capture the photons which passed the breakdown. Photons pass the breakdown region primarily during the first few picoseconds of the laser pulse, when the breakdown is still very small and partly transparent. The plasma expands within a few pico- to nanoseconds, becomes optically thick and absorbs the majority of the incoming photons. This process is described in more detail by Chen et al. [30]. For both positions, the volume absorber measured the average energy over two thousand individual pulses. The energy difference between both positions corresponded to the amount of energy that did not pass the breakdown region. Therefore, this energy fraction either must have been absorbed, diffracted or scattered. Measurements with the volume absorber sideways and below the focal point did not provide indication of any scattering. According to the review paper by Phuoc [7], scattering and diffraction are negligible for laser-induced breakdowns. Therefore, we concluded that, the energy difference virtually equals the pulse energy that was absorbed by the breakdown. The average incident and transmitted pulse energies $\left(E_{\mathrm{P}}\right.$ and $E_{\mathrm{T}}$, respectively) are given in Table 1, along with the calculated absorbed energy $E_{\mathrm{A}}$ and the corresponding standard deviations. $E_{\mathrm{A}}$ was determined to be $121.2 \mathrm{~mJ}$, which is $62.7 \%$ of the incident pulse energy.

\subsection{Blast wave energy}

Blast waves from air breakdowns were recorded with high-speed schlieren imaging. Although the majority of the breakdown emission was blocked by the iris aperture, transmission filters were required to protect the camera sensor from saturation. The applied filters ranged from $\tau=0.05$ to 0.5 . The experiment was repeated several times, and the time lag between the camera start trigger and the Q-switch trigger was sequentially increased by increments of $1 \mu \mathrm{s}$. Three breakdowns were recorded for every time to account for statistical spread. Representative Schlieren images are shown in Figure 3. The origin is located at the focal point. The times give the $50 \%$ effective exposure time, which is the instant when half of the integrated camera gate function has passed. Spherical blast waves are clearly visible as circular density gradients. The breakdown is surrounded by a plume of hot, compressed gas after the detachment of the blast wave. It results from the fast expansion and has a nearly constant diameter of about $8 \mathrm{~mm}$, see the image for $100 \mu \mathrm{s}$ in Figure 3. The blast wave diameters were measured with an in-house Matlab algorithm with an accuracy of $0.1 \mathrm{~mm}$. The dotted curve in Figure 4 is the determined expansion trajectory. Every point is an average of three individual breakdown events. The reproducibility was very good, and therefore no error bars are displayed. The white spot at the origin in Figure 3 is the breakdown plasma, which is visible for approximately $25 \mu \mathrm{s}$. Its brightness prevented recordings earlier than $2.5 \mu \mathrm{s}$. Therefore, the separation of the blast wave from the breakdown could not be observed.

The blast wave energy was determined from the expansion trajectory. The measured trajectory covers the transition phase from a strong blast wave to an acoustic wave. This is well illustrated in Figure 4 by the fact that the trajectory is nearly parallel to the sonic velocity curve (Mach number $\mathrm{Ma}=1$ ) for ambient air. In a previous study [21] we compared the suitability of different blast wave models to laser-induced blast waves in air. The model by Jones [31] gave the best fit to the measured trajectories. It consists of a set of semi-empirical equations, which are: 
$\tau=0.543\left[\left(1+4.61 x(t)^{\frac{5}{2}}\right)^{\frac{2}{5}}-1\right]$

$\tau=c_{0} \frac{t}{r_{0}}$

$x(t)=\frac{r(t)}{r_{0}}$

$r_{0}=\left[\frac{6.25 E_{0}}{B \gamma p_{0}}\right]^{\frac{1}{3}}$

Here, $\tau$ is the non-dimensional time, $t$ is the dimensional time, $r$ is the radius, $r_{0}$ is a reference radius, $c_{0}$ is the sonic velocity, $p_{0}$ is the pressure of the unshocked air, $\gamma$ is the isentropic exponent, and $E_{0}$ is the blast wave energy. $B$ is a geometry parameter, values for different cases are given in [32]. Jones' models was fitted to the measured trajectory with an in-house LabVIEW algorithm, which applied the method of least squares. The sum of the least $\Delta y_{i}^{2}$ is $4.554 \mathrm{~mm}^{2}$, what is a good accuracy. The fit is the dashed grey curve in Figure 4. Jones' model gives a blast wave energy of $96.5 \mathrm{~mJ}$, which is $79.6 \%$ of the absorbed breakdown energy. This is in good agreement with Taylor's statement that a blast wave consumes approximately two third of its source's energy [33]. The remaining breakdown energy $\mathrm{E}_{\mathrm{B}}$ after the blast wave detachment calculates to be $24.7 \mathrm{~mJ}$, which is $12.8 \%$ of the incident laser pulse energy.

\section{Air breakdown}

Laser-induced breakdowns were investigated in static air at ambient conditions, i.e., the air temperature ranged between $23^{\circ}$ and $25^{\circ} \mathrm{C}$, and the air pressure was approximately $964 \mathrm{hPa}$. The investigation included high-speed imaging and emission spectroscopy. Equivalent measurements for spray ignitions are presented in section 5.1. The investigation in this section is of particular relevance for the present study, because it does not incorporate any plasma-droplet interaction or combustion. Therefore, the obtained results are important reference data to separate between plasma effects and combustion processes. A discussion of the similarities and differences between air breakdowns and spray ignitions is provided in section 5 .

\subsection{High-speed imaging}

The sustained camera repetition rate of $250 \mathrm{kHz}$ was not sufficient to capture the very fast development of the laser-induced breakdowns in all details. Therefore, the time lag between the camera start trigger and the Q- 
switch trigger was sequentially increased. Another challenge was the wide dynamic range of the breakdown luminosity. The initial luminosity was very bright, followed by an exponential decrease. Different broadband transmission filters were mounted in front of the camera lens for different times after the laser pulse. Their transmissions ranged from $\tau=0.001$ to 0.5 . Five recordings were performed for every investigated time. The camera exposure time of $1 \mu \mathrm{s}$ was relatively long compared to the transient breakdown morphology, and special efforts were put into the fine adjustment of the time lag between the camera start trigger and the Q-switch trigger. Figure 5 shows a schematic of the camera gate function. The non-dimensional sensitivity is plotted as a function of time. The camera gate features a fast rising edge and a slower falling edge. The overall CMOS sensor exposure time was $1.09 \mu \mathrm{s} \pm 40 \mathrm{~ns}$. To record the later breakdown phases, the Q-switch trigger was sequentially shifted up to several microseconds before the camera exposure. Representative images from this experiment are displayed in Figure 6. The first row illustrates the droplet distribution, which is only relevant in section 5.1. The times give the beginning and the end of the camera exposure. The first two rows of the breakdown sequence show images with exposure start times before the laser pulse. Exposure start times -1.06 $\mu$ s and $0.06 \mu \mathrm{s}$ (second and third row) mean that, the exposure begins before the laser pulse, while the flow channel is still shrouded in darkness. The laser Q-switch time for the exposure start at $-1.06 \mu \mathrm{s}$ is shown in Figure 5. The two rows represent the first $30 \mathrm{~ns}$ and $1.03 \mu \mathrm{s}$ of the breakdown, respectively. The direction of the focused laser beam is from top left to bottom right with a downward angle of 23 degrees. The origins of the images in Figure 6 correspond to the focal point. The three columns show breakdowns during the first $90 \mu \mathrm{s}$ after the laser pulse in ambient air and in spray. They are shown in one figure for a comparison and discussion, which is provided in section 5.1. This section focusses exclusively on the first column of Figure 6.

\subsubsection{Analysis of the transient morphology}

The sequence in the first column of Figure 6 shows a rapid initial growth during the first few microseconds, a subsequent longitudinal expansion, and the generation of three consecutive lobes $(a, b, c)$. All images of the sequence are averages of the recordings from five individual breakdowns. They were created by arithmetical averaging with the image recording and processing software LaVision DaVis 7. The centroid of the breakdown is located to the left of the focal point. This effect is explained by Chen et al. [30]: A breakdown initiates before the focal point when the irradiance of the converging laser pulse exceeds the breakdown threshold. The first lobe (a) expands into the incident direction of the laser beam. The second lobe (b), which is marginally smaller and weaker, is located close to focal point. The right tail of the breakdown features a much smaller and weaker third lobe (c). The first and third lobe decrease quickly. The second lobe develops into a disk-like shape, which can be seen between 27.94 and $29.03 \mu$ s. In total, shrinkage begins after 5 to $6 \mu$ s. We performed a numerical simulation to analyze the flow processes leading to this development. In short, it results from a rollup motion, caused by two counterrotating toroidal vortices. A thorough discussion of the numerical simulation is given by Joarder et al. [22].

The compact shape and the defined surface of the breakdown and its rotational symmetry permit to analyze its transient volume and dimensions. A rotated Cartesian coordinate system with the axes $\eta$ and $\xi$ is introduced, see Figure 7. The origin is located at the focal point. The rotation angle is 23 degrees clockwise, corresponding 
to the downward angle of the laser pulse. The horizontal and vertical expansion in this coordinate system and the volume change of the breakdown were determined with a set of in-house Matlab algorithms. A problem arose from the $1.09 \mu \mathrm{s}$ exposure time of the camera, which is long compared to the time scale of the measurements. It implies that, the sequences in Figure 6 are mathematical convolutions of the exposure time with the time scale of the sequence. Deconvolution by image processing was not possible, because more intermediate time steps would have been necessary. Therefore, the Matlab analysis was performed, and deconvolution was achieved afterwards by the adequate assignment of times for the determined coordinates and volumes: The breakdown grows in all directions during the first few microseconds. The expansion stops, and shrinkage occurs in all directions. Hence, all images taken during the growth phase represent the size at the end of the camera exposure. Accordingly, all images from the shrinkage phase show the size at the beginning of the exposure. Thus, deconvoluted diagrams were achieved by assigning the data from the Matlab analysis either to the start time or the end time of the camera exposure. The results of the spatial analysis are shown as black dots in Figure 8. The error bars represent the standard deviations from five individual breakdowns. Similar results were presented by Kawahara et al. [34] for a laser pulse energy of $170 \mathrm{~mJ}$. They used a $200 \mathrm{~mm}$ lens to focus the laser pulse (we used a $120 \mathrm{~mm}$ lens), resulting in a thinner and longer breakdown. Figure 8a shows the rotationally symmetric breakdown volume. It grows quickly to its maximum size, which is achieved between 4.94 and $5.53 \mu \mathrm{s}$ after the laser pulse. The shrinkage is nearly linear at a significantly smaller rate. The last data point is given $13.94 \mu \mathrm{s}$ after the laser pulse. An analysis beyond this time was not possible due to the degrading signalto-noise ratio of the images. Figure $8 \mathrm{~b}$ shows the coordinates of the breakdown head and tail along the $\xi$-axis. The head is defined as the vertical edge in the laser beam incident direction (negative $\xi$ ). The initial growth along $\xi$ is followed by a linear shrinkage. Figure $8 \mathrm{c}$ shows the top and bottom edges along the $\eta$-axis. Here, no shrinkage is observed after the initial growth phase. The reason is evident from Figure 6 . The second lobe of the breakdown flattens to a disk after $9.94 \mu$ s, but the diameter remains constant.

The spatial development rates were determined by the first derivatives of polynomial fits with the software Origin 9. Polynomials of fourth and fifth order were fitted to the growth phase, while linear functions were fitted to the shrinkage phase. The fitted function are plotted as thin dashed and dotted curves in Figure 8. The velocities are displayed as solid red curves. A comparison of the absolute horizontal and vertical edge velocities is shown in Figure 9, along with Mach numbers for the lab atmosphere. The air temperature and pressure were measured to be $23.1^{\circ} \mathrm{C}$ and $963.6 \mathrm{hPa}$ during the experiment. The humidity was $45.8 \%$. The sonic velocity was calculated to be $345.89 \mathrm{~m} / \mathrm{s}$. Figure 9 shows that, the initial breakdown expansion lies in the supersonic regime. Vertical expansion is around a Mach number of $\mathrm{Ma}=2$, and the horizontal expansion is above $\mathrm{Ma}=3$. Vertical and horizontal expansion become subsonic after $1.3 \mu \mathrm{s}$ and $3 \mu \mathrm{s}$, respectively. The supersonic expansion and its rapid deceleration during the first few microseconds explain well the formation of the spherical blast wave: High initial temperatures of the plasma species of up to $10^{6} \mathrm{~K}$ [27] imply a high overpressure against the lab atmosphere. It results in a supersonic expansion, which creates an LSD wave at the interface between the plasma and the surrounding air [29]. Temperatures reduce to an order of $10^{4}$ to $10^{5} \mathrm{~K}$ after the laser pulse has terminated $[28,30,35]$. Consequently, the pressure decreases and breakdown expansion decelerates. Conservation of momentum causes the detachment of the LSD wave, resulting in a separated blast wave. Beduneau et al. [24] observed the blast wave detachment after $700 \mathrm{~ns}$ for a laser pulse energy of $11.7 \mathrm{~mJ}$. Wang et al. [36] observed 
the detachment after $430 \mathrm{~ns}$ and $750 \mathrm{~ns}$ for a laser pulse length of $33 \mathrm{~ns}$ and pulse energies of $1 \mathrm{~J}$ and $2 \mathrm{~J}$, respectively. Similar to the experiment presented in this study, their breakdowns expanded faster in the longitudinal than in the axial direction. Therefore, blast waves initially have ovoid shapes, which turn spherical after several microseconds. In addition to the blast wave trajectory, Figure 4 shows the front edge and the upper edge trajectories of the breakdown from Figure 8 by two solid grey curves. Together with the blast wave trajectory, they indicate a detachment of the blast wave from the breakdown approximately 1 to $2 \mu$ s after the laser pulse.

\subsubsection{Intensity}

Intensity scales in Figure 6 correspond to the individual minima and maxima of the images and therefore must not be compared between different images. A diagram of the peak intensities from the image sequences is displayed in Figure 10. The diagram shows the intensity curve for both, air breakdown and spray ignition. The latter is discussed in section 5.1. The x-axis gives the $50 \%$ effective exposure times of the images. The rising edge in the beginning is explained as follows: For the first two data points, the laser Q-switch trigger is given during the camera gate. The maximum is reached when the camera start trigger and the Q-switch trigger are given nearly simultaneously. After the maximum, the intensity decreases by three orders of magnitude within $5 \mu \mathrm{s}$. Afterwards, the intensity of air breakdowns decreases steadily for about $15 \mu \mathrm{s}$. A steep decrease begins after $20 \mu \mathrm{s}$, and the intensity falls below the detection limit after $30 \mu \mathrm{s}$. The air breakdown is a pure plasma and its intensity decrease is an indicator for the plasma decay. It extinguishes after approximately $30 \mu \mathrm{s}$.

\subsection{Spectroscopy}

The high-speed imaging sequences provided details of the transient morphology of the air breakdown, but no information regarding the physical processes inside the breakdown plasma. Therefore, a spectroscopic investigation was performed, and the results are presented in this section. The first $40 \mu \mathrm{s}$ after the laser pulse in ambient air were investigated with an exposure time of $50 \mathrm{~ns}$. Multiple exposures were required to obtain a good signal to-noise-ratio. All spectra that are shown and discussed in this section are multiple exposures over one hundred individual breakdowns. The time lag between the spectrometer trigger and the Q-switch trigger was sequentially increased to investigate the breakdowns at different stages of their development. Figure 11 shows a spectroscopic air breakdown sequence during the first $0.6 \mu$ s after the laser pulse. Very high intensities are observed during the first $500 \mathrm{~ns}$, with a maximum between 50 and $100 \mathrm{~ns}$. The spectra show a strong continuum background, which results from bremsstrahlung and indicates a highly ionized plasma. The background is superimposed by strong lines of nitrogen ions $\left(\mathrm{N}_{\mathrm{II}}\right)$. The continuum background decreases rapidly within $500 \mathrm{~ns}$ after the laser pulse. Also the nitrogen ion lines decrease, while atom lines appear. Figure 12 shows two diagrams with five selected spectra for times between 0.1 to $20 \mu \mathrm{s}$. Logarithmic ordinates are used because of the extreme dynamic range. Figure 12a shows three spectra between 0.1 and $1 \mu \mathrm{s}$. The decrease of the continuum background is obvious. Several decreasing $\mathrm{N}_{\mathrm{II}}$ lines are visible between 343 and $571 \mathrm{~nm}$. Atom lines of nitrogen $\left(\mathrm{N}_{\mathrm{I}}\right)$ and oxygen $\left(\mathrm{O}_{\mathrm{I}}\right)$ increase between 742 and $825 \mathrm{~nm}$. Also the $\mathrm{H}_{\alpha}$ hydrogen atom line of the Balmer series at $656.279 \mathrm{~nm}$ is identified, but it is interrupted by a blind spot which is a characteristic of the spectrometer. The hydrogen lines 
originate from air humidity. Figure $12 \mathrm{~b}$ shows two spectra for 5 and $20 \mu \mathrm{s}$. The continuum background has disappeared, ion lines are barely visible. $\mathrm{N}_{\mathrm{I}}$ and $\mathrm{O}_{\mathrm{I}}$ lines feature a high amplitude at $0.5 \mu \mathrm{s}$. Several humps at a low signal-to-noise ratio are visible between 345 and $430 \mathrm{~nm}$ at $20 \mu \mathrm{s}$. They result from the $\mathrm{B}^{2} \Sigma_{\mathrm{u}}{ }^{+}-\mathrm{X}^{2} \Sigma_{\mathrm{g}}{ }^{+}$transition of $\mathrm{N}_{2}{ }^{+}$which forms by recombination processes in the decaying plasma. Five ion and atom lines were analyzed with a set of in-house LabVIEW algorithms. The continuum background was removed, and Lorentz profiles were fitted to the lines with a Levenberg-Marquardt algorithm. Figure 13 shows the amplitudes of the fits to the $\mathrm{N}_{\text {II }}$ ion lines at $343.715,463.054$ and $504.51 \mathrm{~nm}$, and to the $\mathrm{N}_{\mathrm{I}}$ atom lines at 744.229 and $746.831 \mathrm{~nm}$. The intensities of the ion lines increase to a maximum between 110 and $180 \mathrm{~ns}$, which is followed by a decrease of two

orders of magnitude. An exponential decay curve $I(t) / I_{0}=f\left(e^{(-t / \tau)}\right)$ was fitted to the data points of the ion line at $463.054 \mathrm{~nm}$ with Origin 9. The fitted curve features a mean lifetime of $\tau=0.193 \mu \mathrm{s}$. The detection limit is reached after 0.7 to $1.5 \mu \mathrm{s}$. The first atom lines are identified after $220 \mathrm{~ns}$. Their reach their maximum after $1 \mu \mathrm{s}$, which is one order of magnitude weaker than the maximum of the ion lines. Afterwards, the atom lines decrease exponentially. A steep decrease occurs after $13 \mu \mathrm{s}$, and the detection limit is reached after 20 to $22 \mu \mathrm{s}$. The fit of an exponential decay curve to the data points of the atom line at $746.831 \mathrm{~nm}$ revealed a mean lifetime of $\tau=6.30 \mu \mathrm{s}$, which is about thirty times longer than the mean lifetime of the nitrogen ions. The decay curves are indicated by dashed black and white curves in Figure 13. Decay curves were also fitted to ion and atom lines in spray ignition spectra, see section 5.2. A comparison of all mean lifetimes is provided in Table 2 and discussed in section 5.2. Kawahara et al. [34] measured similar spectra for air breakdowns with a laser pulse energy of $170 \mathrm{~mJ}$. They observed a decrease of ion lines and an increase of atom lines between 0.2 and $1 \mu \mathrm{s}$, which is in good agreement with our observations. The maximum of the atom lines is found at a time, when the ion lines have decreased close to the detection limit. This indicates a recombination process of ions with electrons towards atoms. Thus, the plasma state of the air breakdown ends only few microseconds after the laser pulse. A comparison with Figure 6 shows that, the breakdown expands quickly during the plasma stage, and expansion continues until 5 to $6 \mu \mathrm{s}$. The expansion maximum is reached when the ion lines have already disappeared. At this stage, the breakdown primarily consists of dissociated gas.

\section{Spray ignition}

The continuous air co-flow inside the flow channel was at ambient pressure and a temperature of approximately $15^{\circ} \mathrm{C}$ during this investigation. The lower-than-lab-atmosphere temperature resulted from the expansion from a 6-bar in-house supply line. The results from the investigation of air breakdowns, see section 4, are compared to spray ignitions, in order to better understand the interaction of breakdown plasma and fuel droplets.

\subsection{High-speed imaging}

This experiment investigated the first $90 \mu$ s of laser-induced spray ignitions after the laser pulse. The setup and the procedure of the high-speed imaging were the same as in section 4.1. The droplet distribution in the vicinity of the focal point is illustrated in the second and the third image of the first row of Figure 6 . Images were 
taken with a planar Mie scattering setup, which is described in [26]. The second image of the first row (average droplet distribution) was created from 180 individual images, and shows the area around the spray cone edge $20 \mathrm{~mm}$ below the nozzle. The edge is nearly vertical at this distance. The darkening from the left to the right indicates the increasing droplet density. The horizontal position of the focal point was adjusted to be close to the center of the transition region. The third image of the first row shows a representative instantaneous droplet distribution. The spray is dense in the right part of the image. Individual droplets along the laser beam path are present before the focal point. The average spray ignition images (second column) were created from five individual breakdowns by arithmetical averaging with the software LaVision DaVis 7 .

\subsubsection{Image sequences}

The second and third column of Figure 6 show average and individual images of the spray ignition, respectively. Exposure start times $-1.06 \mu$ s and $-0.06 \mu$ s (second and third row) imply that, the exposure overlaps with the instant of the laser pulse, and consequently Mie scattering of laser radiation on dispersed fuel droplets is visible along the beam path $(\mathrm{d}, \mathrm{e})$. The second image (exposure from -0.06 to $1.03 \mu$ s relative to the laser pulse) shows two consecutive lobes along the laser beam path before the focal point $(f, g)$. Regarding its shape and position, the second lobe $(\mathrm{g})$ is very similar to the breakdown in ambient air (a,b,c). The next row (0.44 to $1.53 \mu \mathrm{s})$ shows that, the two lobes have both expanded. The first lobe (f) features a nearly spherical shape with a diameter of approximately $2 \mathrm{~mm}$. The shape and size of the second lobe $(\mathrm{g})$ is still similar to the breakdown in ambient air. The occurrence of the first lobe, which has no counterpart in air, results from local breakdowns which are initiated by fuel droplets. This becomes obvious in the individual breakdown images: The lobe (f) results from a cluster of small breakdowns (h). This effect was also observed by Lawes et al. [12] and Kawahara et al. [15] and is caused by droplets acting as micro-lenses. Fuel droplets before the focal point (d) refocus parts of the laser radiation and create small breakdowns. The reader is referred to the papers by Chýlek et al. and Kawahara et al. for in-depth investigations of this effect [15,37]. The dispersion of fuel droplets in sprays is highly stochastic, and therefore the position, size and shape of the lobe (f) varies between individual breakdowns. A stochastic droplet distribution can have a strong impact on the ignition reliability, as observed by Groß et al. [13] in a reciprocating engine with a very inhomogeneous mixture fraction. But it was insignificant in the present study, as we created breakdowns by abundant laser pulse energy, resulting in an ignition probability of $100 \%$. In the next row $(2.94$ to $4.03 \mu \mathrm{s})$, the spray breakdown differs significantly from the ambient air breakdown. While the three lobes (a,b,c) in ambient are fully developed, the spray breakdown lobes (f) and (g) have merged to an ellipsoid shape with a length of $6 \mathrm{~mm}$ and a diameter of $2 \mathrm{~mm}$. From this time on, the breakdown surface is blurred and fibrous in the individual images. This is very untypical for a decaying breakdown plasma and demonstrates that, the transition from a plasma to a flame kernel has advanced considerably or is completed at this time. The subsequent development of the flame kernel shows the formation of a disk (i). Apart from an unsymmetrical distortion, the disk resembles the air breakdown. This shows that, the same flow process as in air, a rollup motion caused by two counterrotating toroidal vortices, is active. But it is apparently distorted, probably by the presence of a droplet-laden flow field and the heat release from the flame kernel. Finally, the intensity approaches the detectability limit at 88.94 to $90.03 \mu \mathrm{s}$. 


\subsubsection{Intensity}

Intensity scales in Figure 6 correspond to the individual minima and maxima of the images. The diagram in Figure 10 shows the intensity curve for spray ignition as a solid grey curve. Intensities decreases by three orders of magnitude within a few microseconds after the maximum. Between 3 and $20 \mu$ s the intensity of the spray ignition is significantly weaker the intensity of the air breakdown. In contrast, it is still present at later times while the air breakdown disappears after $30 \mu \mathrm{s}$. It decreases only slowly and approaches at the detectability limit after $90 \mu$ s (the zigzag between 10 and $30 \mu$ s results from the usage of different transmission filters in front of the camera lens). Comparing all columns of Figure 6 and the graphs of Figure 10, an important finding from this experiment is the disappearance of the breakdown luminosity after $30 \mu \mathrm{s}$ in air, while breakdowns in two-phase flows are visible for $90 \mu \mathrm{s}$. This gives indication that other processes than plasma radiation are responsible for the luminosity at later times. Chemiluminescence from combustion radicals is an obvious candidate. This is discussed in greater depth in the section 5.2, which presents a spectroscopic investigation of the spray ignition.

\subsection{Spectroscopy}

The first $20 \mu \mathrm{s}$ of the spray ignition were investigated with an exposure time of $100 \mathrm{~ns}$. An exposure time of $100 \mu \mathrm{s}$ was used to capture the emissions from later stages, when intensities have decreased by several orders of magnitude. It was also used at earlier times to obtain spectra with high signal-to-noise ratios. Figure 14 shows two diagrams with five selected spectra for times between 0.1 to $20 \mu \mathrm{s}$ and an exposure time of $100 \mathrm{~ns}$, similar to Figure 12 for air breakdowns. Please note, a direct comparison of the absolute intensities between the Figures 12 and 14 is not possible because of the usage of different collimation optics. The spectra of Figure 14 are multiple exposures over twenty individual ignitions. Logarithmic ordinates are used because of the extreme dynamic range. Figure 14a shows three spectra between 0.1 and $1 \mu$ s. Like in ambient air, the first 500 ns show a bright continuum background, which results from bremsstrahlung. The same ion and atom lines like in air are visible. However, there are some significant differences: First, the $\mathrm{H}_{\alpha}$ Balmer line at $656.279 \mathrm{~nm}$ is stronger, and the $\mathrm{H}_{\beta}$ line at $486.133 \mathrm{~nm}$ is visible. They primarily originate from hydrogen atoms of dissociated fuel molecules. Second, the $\mathrm{CN}^{*} \mathrm{~B}^{2} \Sigma^{+}-\mathrm{X}^{2} \Sigma^{+}$band between 374 and $388.4 \mathrm{~nm}$ appears after $0.2 \mu$ s. Figure $14 \mathrm{~b}$ is significantly different compared to its equivalent, Figure 12b: No atom lines are detected, and the spectra are dominated by the $C^{*} B^{2} \Sigma^{+}-X^{2} \Sigma^{+}$bands and the $C_{2} * d^{3} \Pi_{\mathrm{g}}-\mathrm{a}^{3} \Pi_{\mathrm{u}}$ bands of the Swan system. Several ion and atom lines were analyzed with the same algorithms that were used for the lines in ambient air. Figure 15 shows the amplitudes of Lorentz profile fits to the $\mathrm{N}_{\mathrm{II}}$ lines at 399.500 and $463.054 \mathrm{~nm}$, and to the $\mathrm{N}_{\mathrm{I}}$ lines at 744.229 and $746.831 \mathrm{~nm}$. The maximum of the ion lines lies between 50 and $150 \mathrm{~ns}$, which agrees well with the air breakdowns. It is followed by a rapid decrease by two orders of magnitude and drops below the detection limit after $500 \mathrm{~ns}$. An exponential decay curve was fitted to the data points of the ion line at $463.054 \mathrm{~nm}$ and revealed a mean lifetime of $\tau=0.105 \mu \mathrm{s}$, which is about half as long as the mean lifetime in air breakdowns. The atom lines become visible after $150 \mathrm{~ns}$. The fit of an exponential decay curve to the data points of the atom line at $746.831 \mathrm{~nm}$ revealed a mean lifetime of $\tau=1.56 \mu \mathrm{s}$, which is about four times shorter than the mean lifetime of 
the nitrogen atoms in air breakdowns. The detectability limit is reached after $3.1 \mu \mathrm{s}$. The decay curves are indicated by dashed black curves in Figure 15. The mean lifetimes of ions and atoms and the periods of the decay curve fits are listed in Table 2. The investigation of mean lifetimes shows that both, ion and atom line intensities decrease faster for spray ignitions than for air breakdowns. Nitrogen ion lines decrease two times faster, and nitrogen atom lines decrease four times faster. This gives indication for a nitrogen consumption by recombination processes, including the formation of $\mathrm{CN}^{*}$.

\subsubsection{Plasma-combustion transition}

Systematic variations of the exposure time and its start after the laser pulse were performed to detect the beginning of the $\mathrm{CH}^{*}$ chemiluminescence. $\mathrm{CH}^{*}$, which is a prominent intermediate reactant in hydrocarbon flames, was not identified in the $100 \mathrm{~ns}$ exposures shown in Figure 14. Its strong $\mathrm{A}^{2} \Delta-\mathrm{X}^{2} \Pi$ band ranges from 416 to $440 \mathrm{~nm}$ and has its most distinctive peak at the $\mathrm{Q}$ branch band head at $431.4 \mathrm{~nm}$. A long exposure time of $100 \mu \mathrm{s}$ was required to obtain an evaluable signal. The flow condition and the laser pulse energy were the same as for all other measurements within this study. A strong signal was found for an exposure start $1 \mu$ s after the laser pulse. The spectrum between 428 and $441 \mathrm{~nm}$ is shown as a solid black curve in the insert of Figure 16. The Q branch band head for vibrational quantum numbers $v^{\prime}=v^{\prime \prime}=0$ was identified. The band is much weaker than the bands of $\mathrm{CN}^{*}$ and $\mathrm{C}_{2}{ }^{*}$, and it overlaps with the band of the $(\Delta \mathrm{v}=+2)$-transition of the Swan system. Moreover, it is superimposed by weak lines of the $\mathrm{B}-\mathrm{X}$ transitions of $\mathrm{CN}^{*}$ and $\mathrm{N}_{2}{ }^{+}$. The different intensities of the three species $\mathrm{CN}^{*}, \mathrm{C}_{2}{ }^{*}$ and $\mathrm{CH}^{*}$ raise the question of their origin. Emissions of $\mathrm{CN}^{*}$ are not strong in typical kerosene spray flames. But the bands of $\mathrm{CH}^{*}$ and $\mathrm{C}_{2}^{*}$ are and typically feature a similar order of magnitude, see for example the jet fuel flame spectra shown in [38]. The fact that the detected $\mathrm{CH}^{*}$ band is much weaker than the $\mathrm{C}_{2}$ * Swan bands indicates either a different formation mechanism or a delay in the combustion chain reactions after the ignition.

In order to determine the origin of the detected radicals, a comparative experiment was performed in an inert atmosphere: The air co-flow and atomization air flow were replaced by nitrogen with a purity of 99.998 \%. A spectrum from this experiment is presented in Figure 16, together with its equivalent in air. Both spectra are very similar. The $\mathrm{CN}^{*}$ and the $\mathrm{C}_{2}{ }^{*}$ bands are visible and feature similar intensities in both, air and nitrogen. This gives strong indication of a radical formation by plasma recombination, rather than by combustion chain reactions. The insert in Figure 16 shows the spectrum from the nitrogen atmosphere between 428 and $441 \mathrm{~nm}$ as a solid grey curve. If $\mathrm{CH}^{*}$ is present at all, its emission is much weaker than in air. An edge is marked by a question mark which might be the $\mathrm{Q}$ branch band head. But this signal may also result from a superposition of noise and lines from other species, for example $\mathrm{N}_{2}^{+}$. The comparison of both spectra indicates a primary $\mathrm{CH}^{*}$ formation mechanism that involves the presence of oxygen. In conclusion, we propose that, the decaying breakdown plasma creates a radical pool which initiates combustion chain reactions. The subsequent paragraph explains this in more detail.

Radicals are known to form by recombination processes in decaying breakdown plasma [39]. For instance, laser-induced breakdown spectroscopy experiments on graphite targets show the formation of $\mathrm{C}_{2}{ }^{*}$ by ion- 
ion [40,41] and ion-atom [42] recombination. The herein presented emission spectroscopy experiments showed a formation of $\mathrm{CN}^{*}$ and $\mathrm{C}_{2} *$ by recombination in spray breakdowns, as well: First, the mean lifetime of nitrogen ions and atoms was found to be considerably shorter in spray breakdowns than in air breakdowns, indicating a consumption by recombination processes, see Table 2 . Second, the $\mathrm{CN}^{*}$ and $\mathrm{C}_{2} *$ intensities did not change significantly when the air in the flow channel was replaced by nitrogen. In contrast, the $\mathrm{CH}^{*}$ radical was only identified in igniting spray in air. According to the present knowledge $[43,44]$, one of the main $\mathrm{CH}^{*}$ formation paths in hydrocarbon flames is $\mathrm{C}_{2}+\mathrm{OH} \rightarrow \mathrm{CH}^{*}+\mathrm{CO}$ [45]. The mechanism requires the reactants $\mathrm{C}_{2}$ and $\mathrm{OH}$ in the ground state. An excited state $\mathrm{AB}^{*}$ can transfer to the ground state $\mathrm{AB}$ by photon emission $\left(\mathrm{AB}^{*} \rightarrow \mathrm{AB}+\mathrm{h} v\right)$ and by collisional quenching $\left(\mathrm{AB}^{*}+\mathrm{M} \rightarrow \mathrm{AB}+\mathrm{M}\right)$. Apart from $\mathrm{C}_{2}$, which originates from excited $\mathrm{C}_{2}{ }^{*}$, the mechanism requires the reactant $\mathrm{OH}$, which was not identified in the experiment. The distinctive $\mathrm{OH}^{*}$ bands lie in the near ultraviolet spectrum, which was inaccessible with the optical setup. But $\mathrm{OH}^{*}$ radicals are frequently observed in different plasmas [46-49]. We identified atomic oxygen and hydrogen, and thus $\mathrm{OH}^{*}$ can form by recombination processes. We propose $\mathrm{CH}^{*}$ to form from the radical pool of the decaying breakdown plasma. The $\mathrm{CH}^{*}$ formation path which is known from hydrocarbon flames may be active here, since the required reactants are very likely to form from the radical pool.

\subsubsection{Rovibrational temperatures}

Breakdown temperatures during the transition from a plasma to a flame kernel are crucial information, in particular for the numerical modelling of two-phase flow ignitions. The $\mathrm{CN}^{*}$ band between 374 and $388.4 \mathrm{~nm}$ features strong intensities between 0.6 and $10 \mu \mathrm{s}$ after the laser pulse. Therefore, it was used to estimate the rovibrational breakdown temperatures during the transition phase. Simulated $\mathrm{CN}^{*}$ bands were fitted to the measured spectra between 384.2 to $388.4 \mathrm{~nm}$ with the software LIFBASE 2.1 [50] by the method of least chi-squares. The selected spectral range covers the $\mathrm{P}$ branches of the band. The Fortrat parabolas of the $\mathrm{P}$ branches have turning points towards the longer wavelengths. Therefore, they feature distinctive peaks which provide a very good signal-to-noise ratio. Figure 17 shows an example of a measured spectrum (in red at the upper half) and of a fitted simulation (in blue at the lower half). The residual is shown as a grey curve along the horizontal axis. The resulting temperatures are given in Figure 18. Spectra with an exposure time of $100 \mathrm{~ns}$ covered the period from 0.6 to $10 \mu \mathrm{s}$. No analyses were performed for earlier times, because the population density distributions of the molecular states were not in a thermal equilibrium. Due to the progressing transition to a flame kernel, the $\mathrm{CN}^{*}$ signal-to-noise ratio decreased and was very low after $10 \mu \mathrm{s}$. Consequently the period from 50 to $150 \mu \mathrm{s}$ was covered by a $100 \mu$ s exposure. The temperatures in the decaying plasma before $2 \mu$ s are around $7800 \mathrm{~K}$. The scattering of the data points before $1.2 \mu$ s probably results from a still incomplete thermal equilibrium of the involved molecular states. Afterwards, temperatures decrease steadily to $6210 \mathrm{~K}$ at $10 \mu \mathrm{s}$. An accuracy analysis of the measured temperatures was performed and revealed an accuracy of $\pm 100 \mathrm{~K}$ for the period from 0.75 to $3 \mu \mathrm{s}$. The incomplete thermodynamic equilibrium at earlier times and the low signal-to-noise-ratio at later times drop the accuracy to $\pm 250 \mathrm{~K}$. An average $\mathrm{CN}^{*}$ radical temperature of $4490 \mathrm{~K}$ was determined for the $100 \mu \mathrm{s}$ exposure from 50 to $150 \mu \mathrm{s}$, which is in good agreement with the temperature decrease of the $100 \mathrm{~ns}$ exposures. 
The temperatures are in reasonable agreement with the measurements in laser-induced air breakdowns by Yalçin et al. [51], Phuoc and White [28] and Kawahara et al. [34]. They independently found temperatures of 10,000 to $20,000 \mathrm{~K}$ for the period between 1 and $2 \mu \mathrm{s}$ after the laser pulses. Our measurements show the same order of magnitude during the plasma stage before $2 \mu$ s and a temperature decrease of the evoking flame kernel towards typical hydrocarbon flame temperatures.

\section{Summary and conclusions}

Non-resonant laser-induced breakdowns were created by focused laser radiation from a frequency-doubled and Q-switched Nd:YAG laser in ambient air and in a kerosene spray. The breakdown energy in air was determined by the measurement of the absorbed laser pulse energy and the energy fraction that was consumed by the blast wave. The absorbed laser pulse energy was measured with a laser power volume absorber before and behind the focal point. The blast wave energy was estimated by the measurement of the expansion trajectory with schlieren imaging and the application of a blast wave model to the trajectory. It was found that, from an average laser pulse energy of $193.1 \mathrm{~mJ}$ only a small fraction of $24.7 \mathrm{~mJ}$ remained in the air breakdown. The breakdown energy in fuel spray was not measured, but it was somewhat smaller, since a fraction of the laser pulse energy was lost by Mie scattering on fuel droplets. The initial breakdown phase was characterized by a plasma state. Optical emission spectroscopy showed that, a continuum background and nitrogen ion lines dominated the spectra of air and spray breakdowns during the first $0.5 \mu$ s after the laser pulse. High-speed imaging of the air breakdowns showed that, the hot plasma expanded at supersonic velocity during the first three microseconds after the laser pulse. The rapid expansion created a shock layer at the interface to the surrounding air. Temperature and pressure decreased after the termination of the laser pulse, which in turn decelerated the expansion. Consequently, conservation of momentum caused the detachment of the shock layer, resulting in a separated blast wave.

The transient morphologies of air breakdowns and kerosene spray ignitions were compared by means of high-speed imaging. Air breakdowns constantly had a compact shape with a defined surface. They became invisible for the applied optical setup after $30 \mu \mathrm{s}$. Their decay was accompanied by shrinkage and the formation to a disk. In contrast, the spray breakdowns became blurred and fibrous after 3 to $4 \mu \mathrm{s}$. They did not shrink but deformed, and their intensities decreased steadily. They became invisible after $90 \mu \mathrm{s}$, which is three times later than the air breakdowns. Their fibrous shapes indicated the evoking flame kernels, and the bright plasma emissions were replaced by a much weaker combustion chemiluminescence. Small breakdowns along the laser beam path occurred in spray due to a micro-lens effect of the present fuel droplets.

The plasma-flame kernel transition in spray was further investigated with optical emission spectroscopy. Recombination reactions to atoms and later to diatomic radicals occurred inside the decaying spray breakdown plasma. These radicals were not detected in air breakdowns. Another significant difference between air breakdowns and spray ignitions were the mean lifetimes of ion and atom lines. In spray ignitions, mean lifetimes of nitrogen ions were two times shorter, and mean lifetime of nitrogen atom lines were four times shorter. Ion lines disappeared after $0.5 \mu \mathrm{s}$ and atom lines disappeared after $3 \mu \mathrm{s}$, indicating a consumption by recombination processes, including the formation of $\mathrm{CN}^{*}$. The time scale is in very good agreement with the rise of the blurred 
and fibrous shapes. In conclusion, the transition from a breakdown plasma to a flame kernel proceeded within few microseconds after the laser pulse.

A comparative spectroscopic investigation of the first $100 \mu$ s after the laser pulse was performed on spray breakdowns in air and in nitrogen. While the spray ignited in air, no ignition was possible in nitrogen. Strong bands of $\mathrm{C}_{2} *$ and $\mathrm{CN}^{*}$ radicals were verified in both atmospheres. The band intensities were virtually equal, verifying that both species form by recombination processes. In contrast, a band of the $\mathrm{CH}^{*}$ radical was only verified in spray ignitions in air but not in spray breakdowns in nitrogen. It indicated that, $\mathrm{CH}^{*}$ is formed by a combustion chain reaction, rather than by plasma recombination. We propose $\mathrm{CH}^{*}$ to form by a reaction path similar to hydrocarbon flames. The required reactants originate from the radical pool which forms in the decaying breakdown plasma through recombination processes.

Spray breakdown temperatures were determined by the fits of simulated $\mathrm{CN}^{*}$ radical bands to the measured bands. Rovibrational temperatures were determined to be around $7800 \mathrm{~K}$ between 0.6 and $2 \mu \mathrm{s}$ after the laser pulse. Temperatures decrease steadily to $6210 \mathrm{~K}$ between 1.2 and $10 \mu \mathrm{s}$. An average temperature of $4490 \mathrm{~K}$ was measured for the period from 50 to $150 \mu \mathrm{s}$.

The present study supports a better understanding of the flame kernel formation in sprays. We strived for high temporal and spatial resolutions, which is very challenging in this difficult environment. But the process is complex and further research is required, before all aspects are well understood. The authors hope that their study gives impulses for future research, which has to incorporate the fields of combustion diagnostics, kinetics and numerical simulation. In particular, a better understanding of the diffraction and displacement of fuel droplets in the immediate vicinity of the breakdown is required. This is a very difficult experimental challenge due to the high irradiance of the breakdown plasma. Also, the development of the radical intensities for times later than $100 \mu$ s can advance the understanding of the plasma-combustion transition, since $\mathrm{CN}^{*}$ should decay while $\mathrm{CH}^{*}$ and $\mathrm{C}_{2} *$ should meet at a similar intensity level once the transition is completed. Such a measurement was not possible in the flow channel, because it featured a steady downward flow of both, air and fuel droplets, which dragged the evoking flame kernel out of the field-of-view of the optics. We suggest that future experiments shall be done in an aerosol chamber with a spectrometer or a photomultiplier. Numerical simulations can provide essential insights, and our results may be used for the numerical modelling of flame kernel generation in twophase flows.

\section{Acknowledgement}

This study received funding from the German Federal Ministry of Economics and Technology through the Project LuFo IV - EffMaTec (reference number 20T0802).

\section{References}

[1]

European Aviation Safety Agency, Certification Specifications for Engines, Specification CS-E 910, Amendment 3, 2010. 
[2]

W. Lazik, T. Doerr, S. Bake, R. v.d. Bank, L. Rackwitz, Development of Lean-Burn Low-NO ${ }_{x}$ Combustion Technology at Rolls-Royce Deutschland, Paper GT2008-51115, Proc. ASME Turbo Expo, Berlin, Germany, 2008.

$[3]$

M.J. Foust, D. Thomsen, R. Stickles, C. Cooper, W. Dodds, Development of the GE Aviation Low Emissions TAPS Combustor for Next Generation Aircraft Engines, Paper AIAA 2012-0936, Proc. 50 ${ }^{\text {th }}$ AIAA Aerosp. Sci. Meeting, Nashville, TN, 2012.

[4]

P.D. Ronney, Opt. Eng. 33 (1994) 510-521.

$[5]$

P.D. Maker, R.W. Terhune, C.M. Savage, Optical third harmonic generation, Proc. $3^{\text {rd }}$ Int. Conf. Quant. Electr., Paris, France, 1963.

[6]

J.H. Lee, R. Knystautas, AIAA J. 7 (1969) 312-317.

[7]

T.X. Phuoc, Opt. and Lasers in Eng. 44 (2006) 351-397.

$[8]$

R. Oldenborg, J. Early, C. Lester, Advanced Ignition and Propulsion Technology Program, Report No. LA-UR98-2272, Los Alamos National Laboratory, 1998.

[9]

G. Kroupa, G. Franz, E. Winkelhofer, Opt. Eng. 48 (2009) 014202.

[10]

N. Pavel, M. Tsunekane, T. Taira, Opt. Expr. 19 (2011) 9378-9384.

A.P. Yalin, Opt. Expr. 21 (2013) A1102-A1112.

[12]

M. Lawes, Y. Lee, A.S. Mokhtar, R. Woolley, Combust. Sci. Tech. 180 (2008) 296-313. 
[13]

V. Groß, H. Kubach, U. Spicher, R. Schieß1, U. Maas, Influence of Laser-induced Ignition on Spray-Guided Combustion - Experimental Results and Numerical Simulation of Ignition Processes, SAE Tech. Paper 200901-2623, 2009.

[14]

C.L. Genzale, L.M. Pickett, A.A. Hoops, J.M. Headrick, Laser Ignition of Multi-Injection Gasoline Sprays, SAE Tech. Paper 2011-01-0659, 2011.

[15]

N. Kawahara, K. Tsuboi, E. Tomita, Opt. Expr. 22 (2014) A44-A52.

[16]

H. El-Rabii, G. Gaborel, J.-P. Lapios, D. Thévenin, J.C. Rolon, J.-P. Martin, Opt. Commun. 256 (2005) 495506.

[17]

S. Barbosa, P. Scouflaire, S. Ducruix, G. Gaborel, Comparison Between Spark Plug and Laser Ignition in a Gas Turbine Combustor, Proc. $3^{\text {rd }}$ European Combust. Meeting (2007).

K.G. Moesl, K.G. Vollmer, T. Sattelmayer, J. Eckstein, H. Kopecek, J. Eng. Gas Turb. Power 131 (2009) 021501 .

[19]

C. Manfletti, G. Kroupa, Opt. Expr. 21 (2013) A1126-A1139.

[20]

G.C. Gebel, S. Le Brun, T. Mosbach, W. Meier, M. Aigner, J. Eng. Gas Turb. Power 135 (2013) 021505.

[21]

G.C. Gebel, T. Mosbach, W. Meier, M. Aigner, Laser-induced Blast Waves in Air and their Effect on Monodisperse Droplet Chains of Ethanol and Kerosene, Paper 0246-000098, Proc. $29^{\text {th }}$ Int. Symp. Shock Waves, Madison, WI, 2013.

[22]

R. Joarder, G.C. Gebel, T. Mosbach, Int. J. Heat Mass Transf. 63 (2013) 284-300. 
[23]

A.H. Lefebvre, D.R. Ballal, Gas Turbine Combustion, $3^{\text {rd }}$ edition, CRC Press, Boca Raton, 2010.

[24]

J.-L. Beduneau, N. Kawahara, T. Nakayama, E. Tomita, Y. Ikeda, Combust. Flame 156 (2009) 642-656.

[25]

C. Letty, E. Mastorakos, A.R. Masri, M. Juddoo, W. O’Loughlin, Exp. Therm. Fl. Sci. 43 (2012) 47-54.

[26]

G.C. Gebel, T. Mosbach, W. Meier, M. Aigner, Experimental Investigation of the Ignition and Flame Stabilization of a Full Cone Kerosene Spray in a Lab-scale Model Combustor, Paper 1204, Proc. $12^{\text {th }}$ Int. Conf. Liq. At. Spray Syst., Heidelberg, Germany, 2012.

[27]

C. Grey-Morgan, Rep. Prog. Phys. 38 (1975) 621-665.

[28]

T.X. Phuoc, F.P. White, Proc. Combust. Inst. 29 (2002) 1621-1628.

[29]

R.G. Root, in: L.J. Radziemski, D.A. Cremers (Eds.), Laser-Induced Plasmas and Application, Marcel-Dekker, New York, 1989, pp. 88-91.

[30]

Y.-L. Chen, J.W.L. Lewis, C. Parriger, J. Quant. Spectrosc. and Rad. Transf. 67 (2000) 91-103.

[31]

D.L. Jones, Phys. Fluids 11 (1968) 1664-1667.

[32]

D.L. Jones, Phys. Fluids 5 (1962) 637.

[33]

G. Taylor, Proc. R. Soc. Lond. A 201 (1950) 159-174.

[34]

N. Kawahara, J.-L. Beduneau, T. Nakayama, E. Tomita, Y. Ikeda, Appl. Phys. B 86 (2007) 605-614. 
T.A. Spiglanin, A. McIlroy, E.W. Fournier, R.B. Cohen, J.A. Syage, Combust. Flame 102 (1995) 310-328.

[36]

B. Wang, K. Komurasaki, T. Yamaguchi, K. Shimamura, Y. Arakawa, J. Appl. Phys. 108 (2010) 124911.

[37]

P. Chýlek, M.A. Jarzembski, V. Srivastava, R.G. Pinnick, J.D. Pendleton, J. Cruncleton, Appl. Opt. 26 (1987) 760-762.

[38]

T. Mosbach, G.C. Gebel, P. le Clercq et al., Investigation of GTL-like Jet Fuel Composition on GT Engine Altitude Ignition and Combustion Performance Part II, Paper GT2011-45510, Proc. ASME Turbo Expo, Vancouver, Canada, 2011.

[39]

R.E. Russo, X.L. Mao, J.H. Yoo, J.J. Gonzales, in: J.P. Singh, S.N. Thakur (Eds.), Laser-Induced Breakdown Spectroscopy, Elsevier, Amsterdam, Oxford, 2007, pp. 49-82.

[40]

K.F. Al-Shoboul, S.S. Harilal, A. Hassanein, Appl. Phys. Lett. 99 (2011) 131506.

[41]

S.S. Harilal, A. Hassanein, M. Polek, J. Appl. Phys. 110 (2011) 053301.

[42]

A. Kushwaha, R.K. Thareja, Appl. Opt. 47 (2008) G65-G71.

[43]

V.N. Nori, Modeling and Analysis of Chemiluminescence Sensing for Syngas, Methane and Jet-A Combuston, Doctoral thesis, Georgia Institute of Technology, Atlanta, 2008.

[44]

T. Kathrotia, Reaction Kinetics Modeling of $\mathrm{OH}^{*}, \mathrm{CH}^{*}$, and $\mathrm{C}_{2}{ }^{*}$ Chemiluminescence, Doctoral thesis, Ruprecht-Karls-Universität, Heidelberg, 2011.

[45]

A.G. Gaydon, The spectroscopy of flames, Wiley, New York, 1974. 
[46]

B. Benstaali, P. Boubert, B.G. Cheron, A. Addou, J.L. Brisset, Plasma Chem. Plasma Proc.22 (2002) 553-571.

[47]

R. Ono, T. Oda, J. Phys. D: Appl. Phys. 41 (2008) 035204.

[48]

N. Srivastava, C. Wang, T.S. Dibble, Eur. Phys. J. D 54 (2009) 77-86.

[49]

P. Bruggeman, D.C. Schram, Plasma Sources Sci. Tech. 19 (2010) 045025.

[50]

J. Luque, D.R. Crosley, LIFBASE: Database and spectral simulation (version 1.5), Report No. MP 99-009, SRI International, 1999.

[51]

Ş. Yalçin, D.R. Crosley, G.P. Smith, G.W. Faris, Appl. Phys. B 68 (1999) 121-130.

TABLES AND FIGURES (This section supports the correct assignment of subscripts to figures and tables. It must be removed from the document for the final version)

Tab. 1 Laser pulse energies in front and behind the breakdown, and the resulting breakdown energy. Average over two thousand laser pulses.

\begin{tabular}{lccc}
\hline & $E_{\mathrm{P}}$ & $E_{\mathrm{T}}$ & $E_{\mathrm{A}}$ \\
\hline Average energy $[\mathrm{mJ}]$ & 193.1 & 71.9 & 121.2 \\
Standard deviation $[\mathrm{mJ}]$ & 1.74 & 1.07 & 2.04 \\
\hline
\end{tabular}

Tab. 2 Mean nitrogen ion and atom line lifetimes in decaying laser-induced breakdowns, and the periods of the exponential decay fits.

\begin{tabular}{lcc}
\hline & $\mathrm{N}_{\mathrm{II}}$ & $\mathrm{N}_{\mathrm{I}}$ \\
& $463.054 \mathrm{~nm}$ & $746.831 \mathrm{~nm}$ \\
\hline$\tau$ in air $[\mu \mathrm{s}]$ & 0.193 & 6.30 \\
$t_{\text {start }} / t_{\text {end }}$ in air $[\mu \mathrm{s}]$ & $0.2 / 0.5$ & $1 / 13$ \\
$\tau$ in spray $[\mu \mathrm{s}]$ & 0.105 & 1.56 \\
\hline
\end{tabular}




\begin{tabular}{lll}
\hline$t_{\text {start }} / t_{\text {end }}$ in spray [ $\left.\mu \mathrm{s}\right]$ & $0.2 / 0.45$ & $0.4 / 1.5$ \\
\hline
\end{tabular}

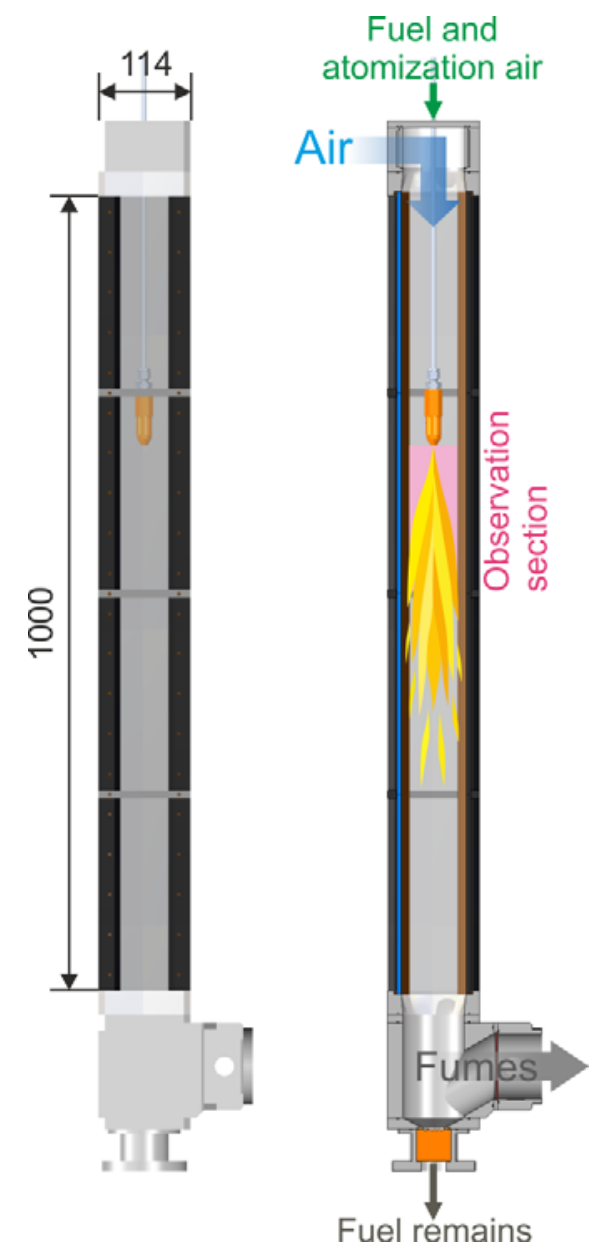

Fig. 1 Schematic representation of the flow channel with the spray nozzle. Scales are given in millimeters.

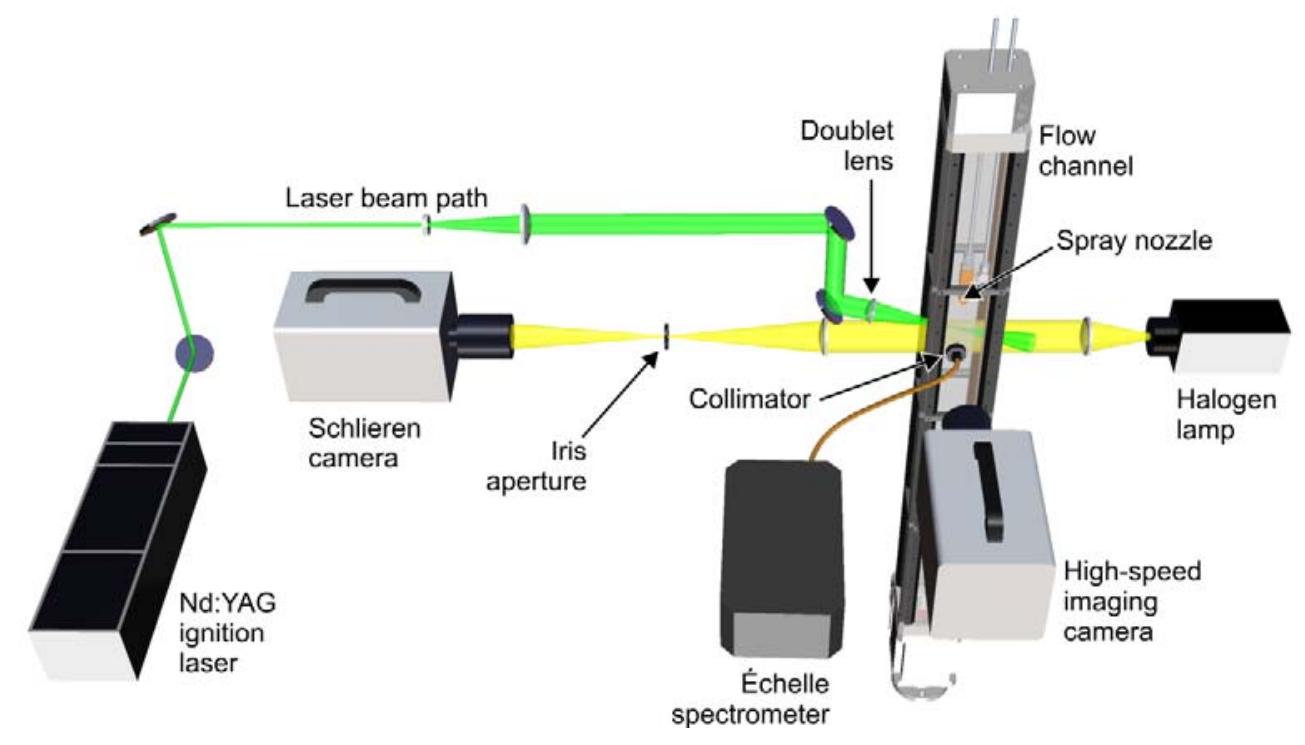


Fig. 2 Schematic representation of the schlieren imaging, high-speed imaging and optical emission spectroscopy setup. Please note, the setups were not applied simultaneously.

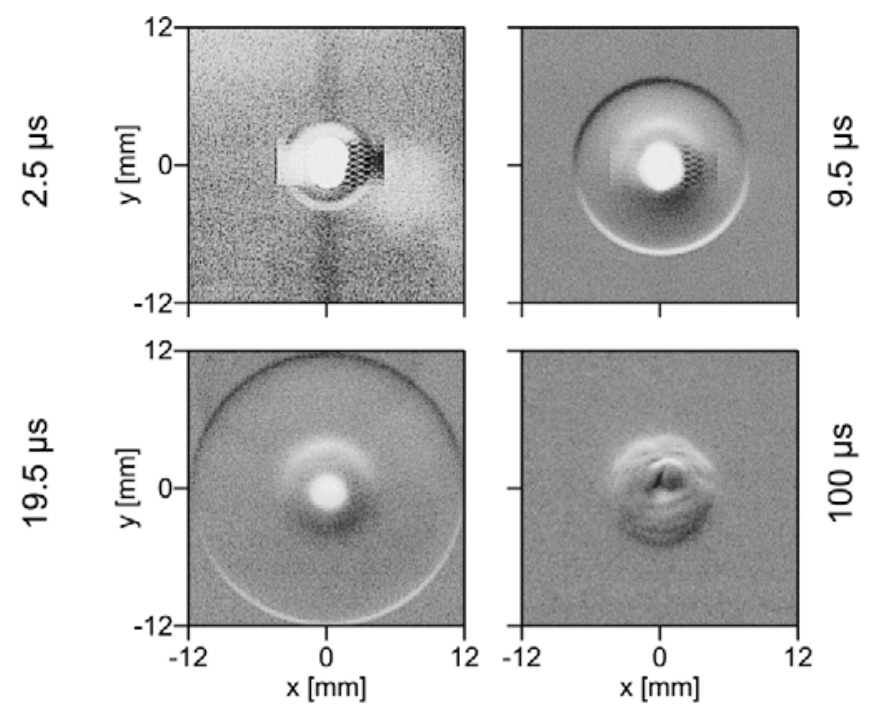

Fig. 3 Schlieren images of a laser-induced air breakdown. The spherical blast wave is visible in the first three images. The white spot at the origin is the breakdown plasma. At $100 \mu \mathrm{s}$ the plasma has disappeared, a gas plume remains.

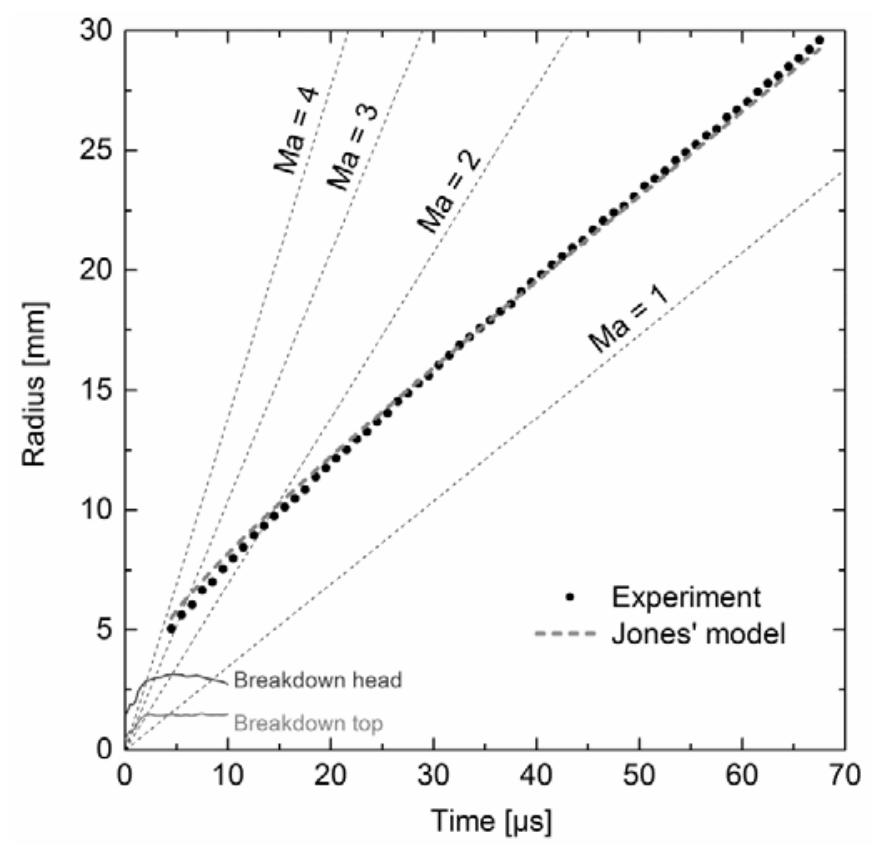

Fig. 4 Comparison of the measured blast wave trajectory with Jones' blast wave model in ambient air. The distance of the air breakdown head and top from the focal point are indicated by two small curves in the lower left corner. They are identical to the curves displayed in Figure 8. 


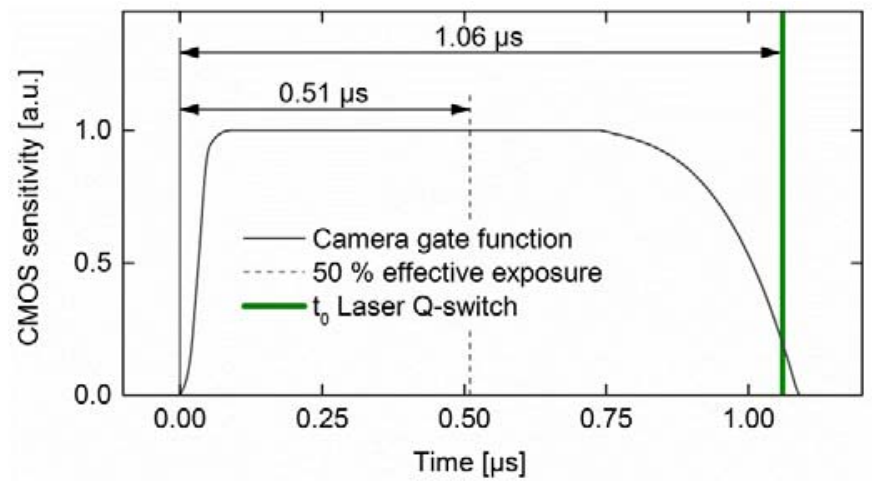

Fig. 5 Camera gate function for an exposure time of $1.09 \mu \mathrm{s}$ and an exposure start $1.06 \mu \mathrm{s}$ before the laser pulse (Q-switch). This diagram illustrates that, the exposure captures only the first $30 \mathrm{~ns}$ of the breakdown. This is the time setting for the images shown in the second row of Figure 6. 

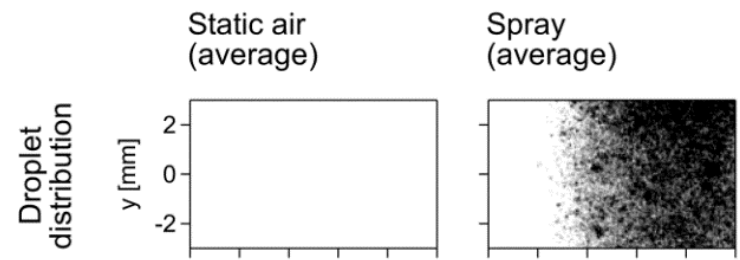

Spray

(individual)
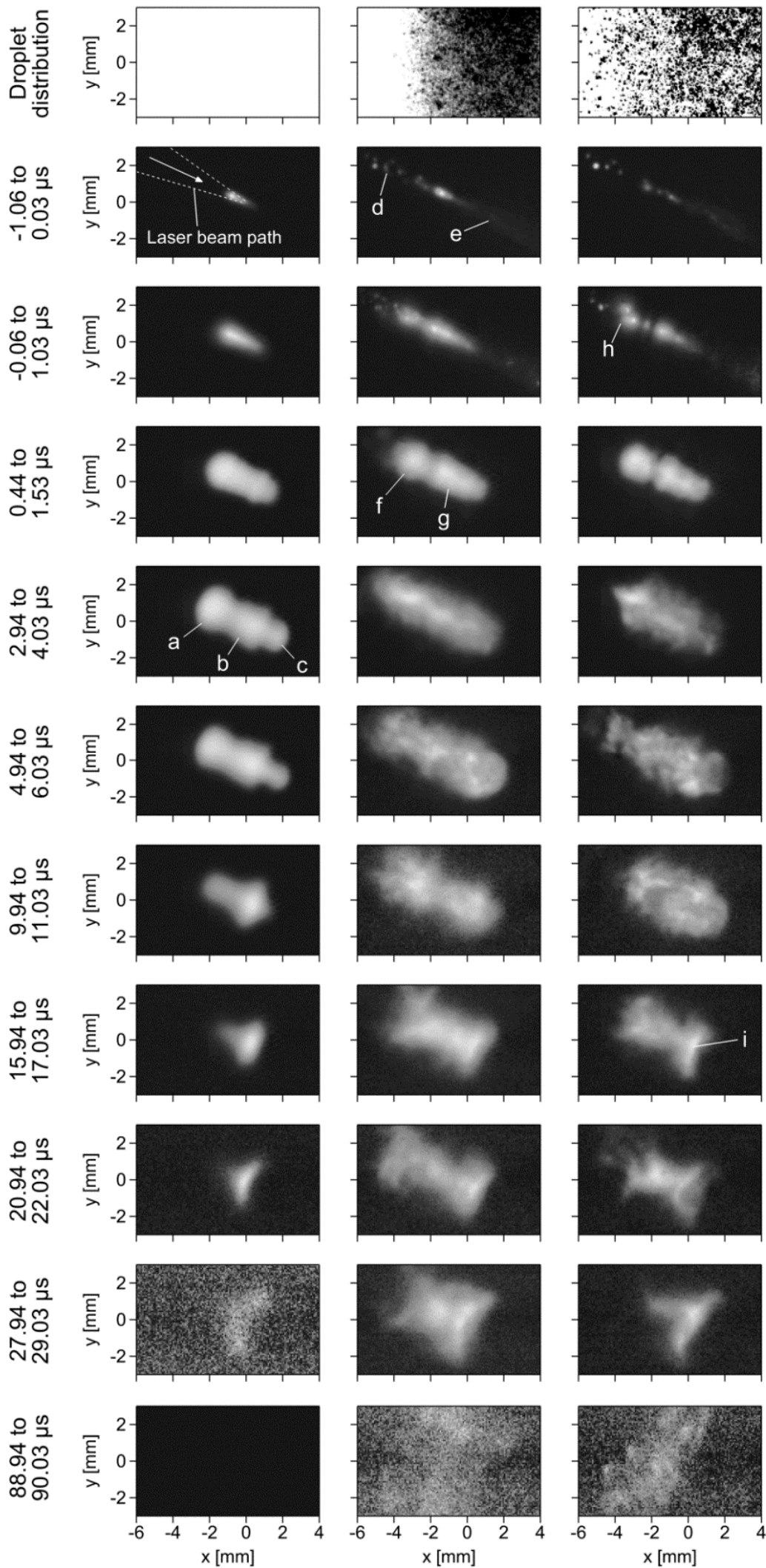
Fig. 6 Sequences of the temporal development of laser-induced air breakdowns (left colum) and kerosene spray ignitions (center and right colums). The first row indicates the droplet distribution of the spray. The times give the beginning and the end of the camera exposure. The center column shows arithmetic averages over five individual ignitions.

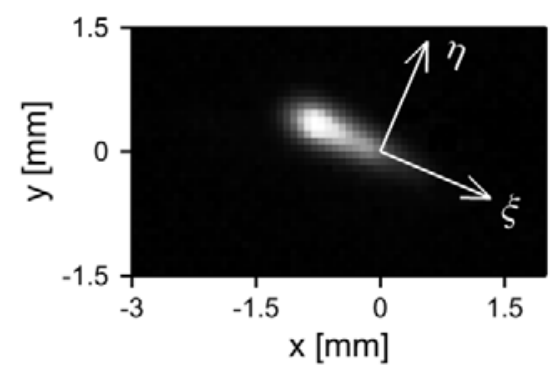

Fig. 7 Air breakdown and a Cartesian coordinate system rotated by $23^{\circ}$ clockwise towards the horizontal axis. The origin is at the focal point.

a)

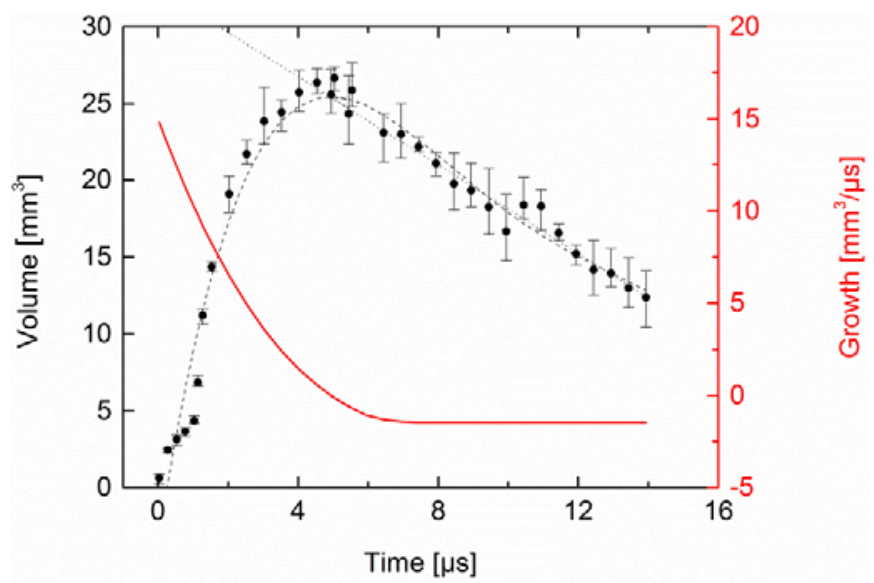


b)

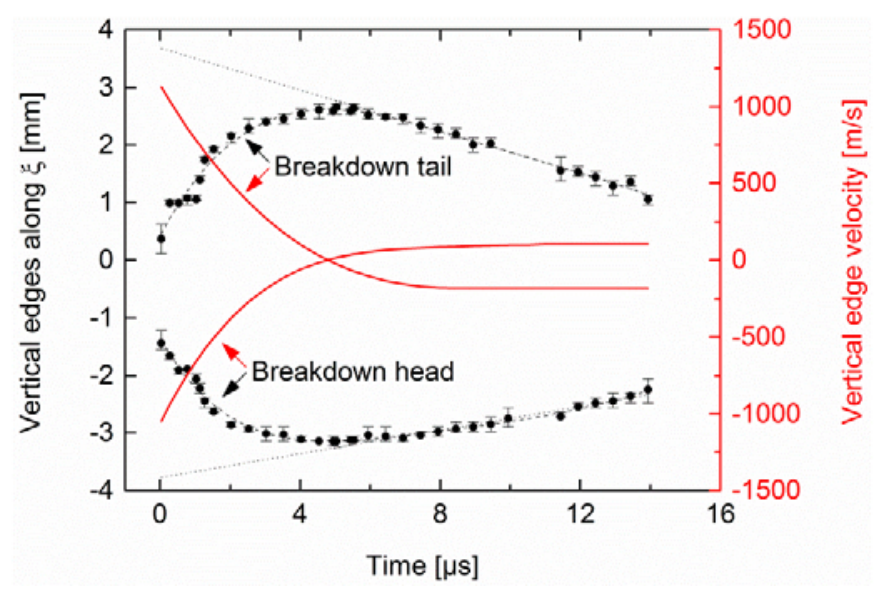

c)

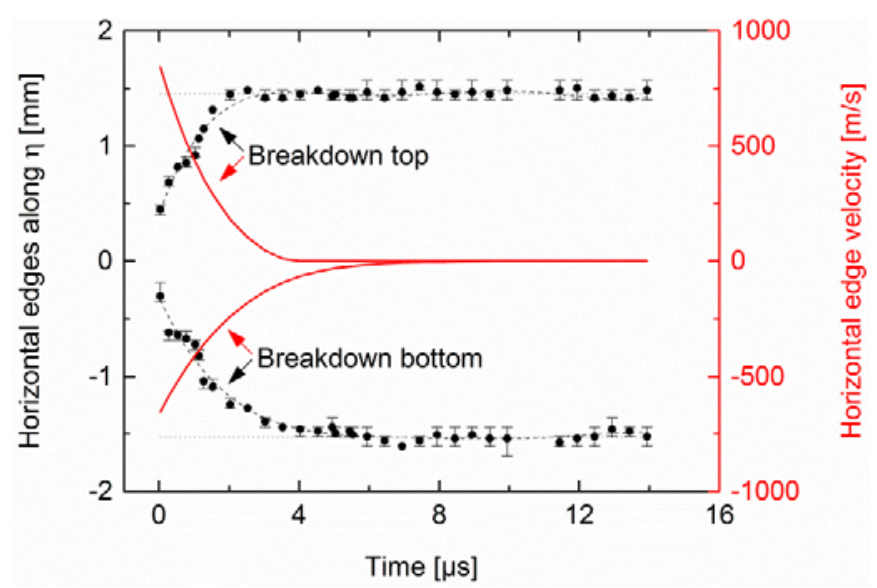

Fig. 8 Transient morphology of the air breakdowns: a) Volume and volumetric growth, b) vertical edge trajectory and velocity, and c) horizontal edge trajectory and velocity. The breakdown head is at the laser beam incident side. 


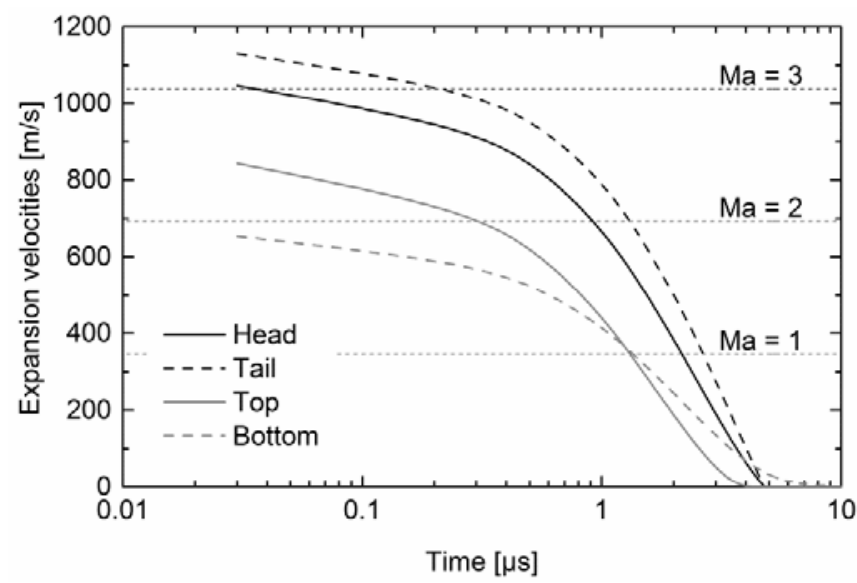

Fig. 9 Absolute velocities of the air breakdown edges. The Mach numbers are given for the undisturbed lab atmosphere.

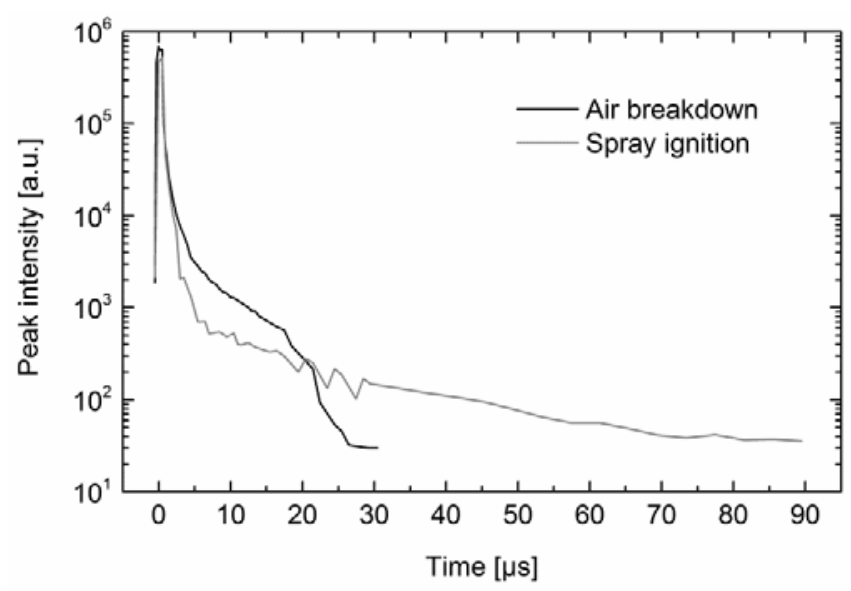

Fig. 10 Peak intensities of the recorded images for laser-induced air breakdowns and spray ignitions. The initial intensities of the air breakdowns are higher, but they disappear after $30 \mu \mathrm{s}$. In contrast, the spray ignition intensities are detected for $90 \mu \mathrm{s}$. 


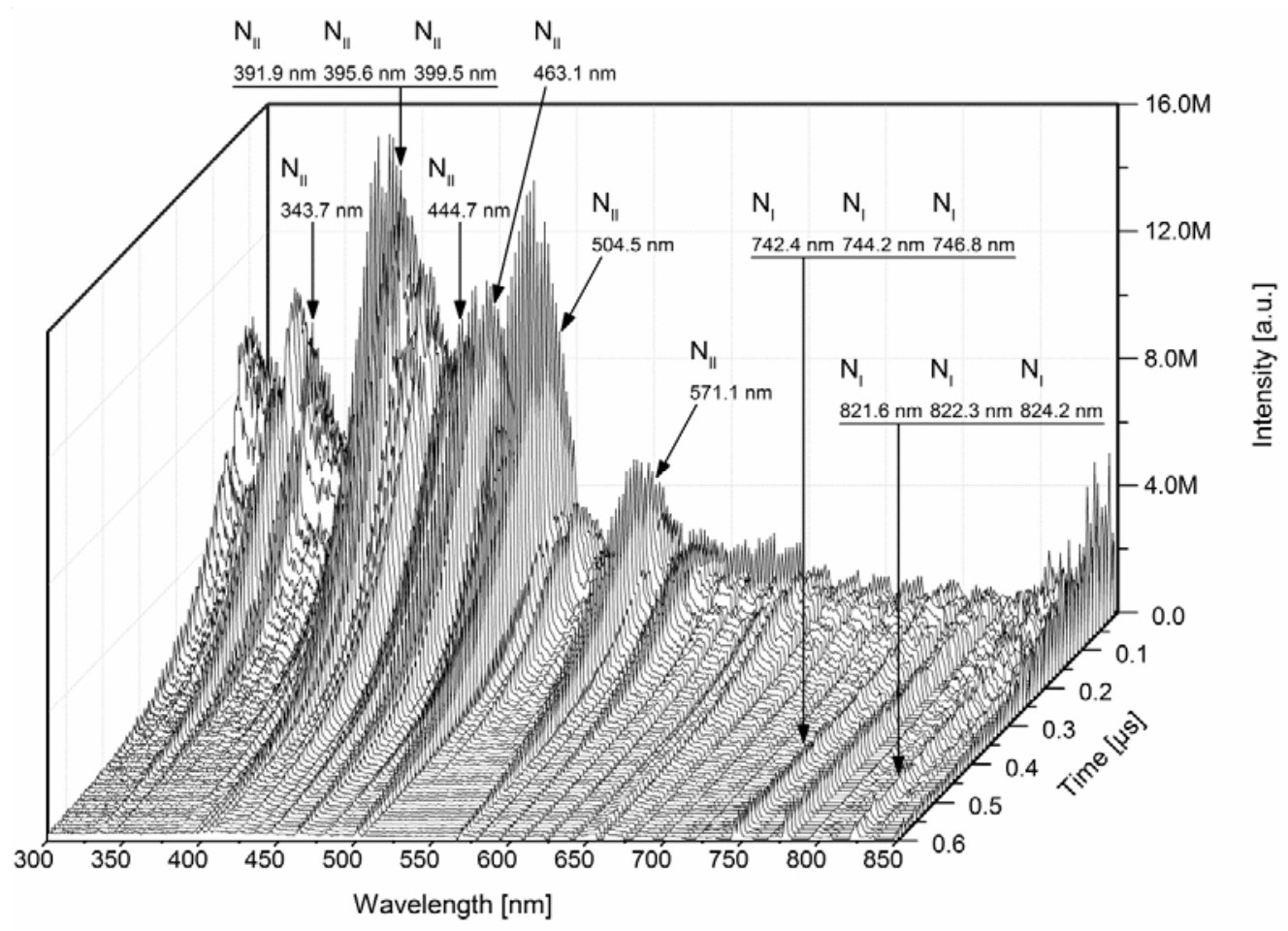

Fig. 11 Ensemble of the optical emission spectra from laser-induced air breakdowns. Every spectrum is an overlay of one hundred individual breakdowns. The exposure time for each spectrum is $50 \mathrm{~ns}$.

a)

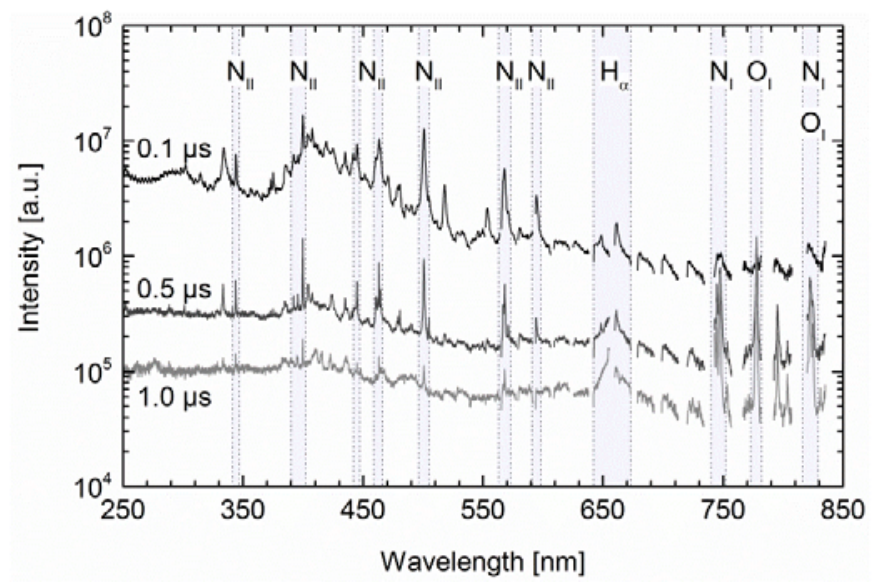


b)

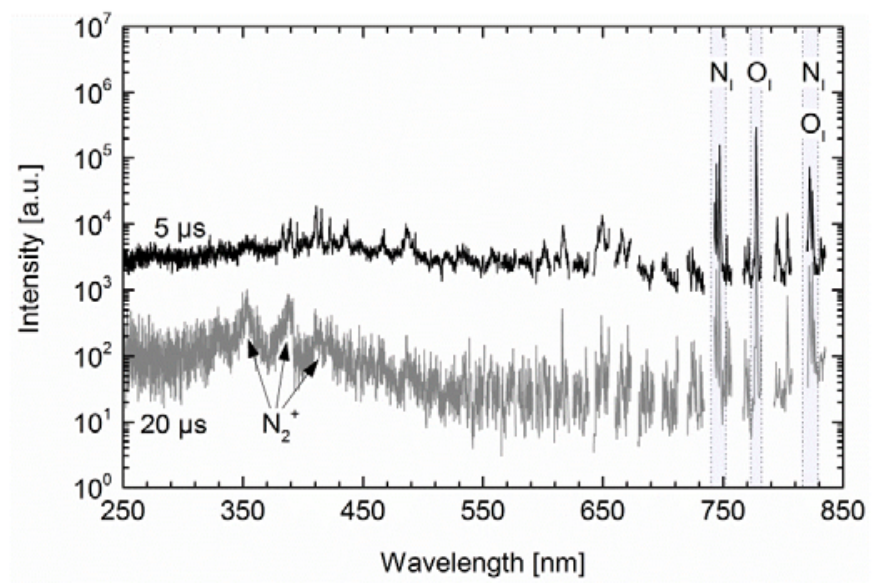

Fig. 12 Optical emission spectra from laser-induced air breakdowns. Every spectrum is an overlay of one hundred individual breakdowns. The exposure time for each spectrum is $50 \mathrm{~ns}$.

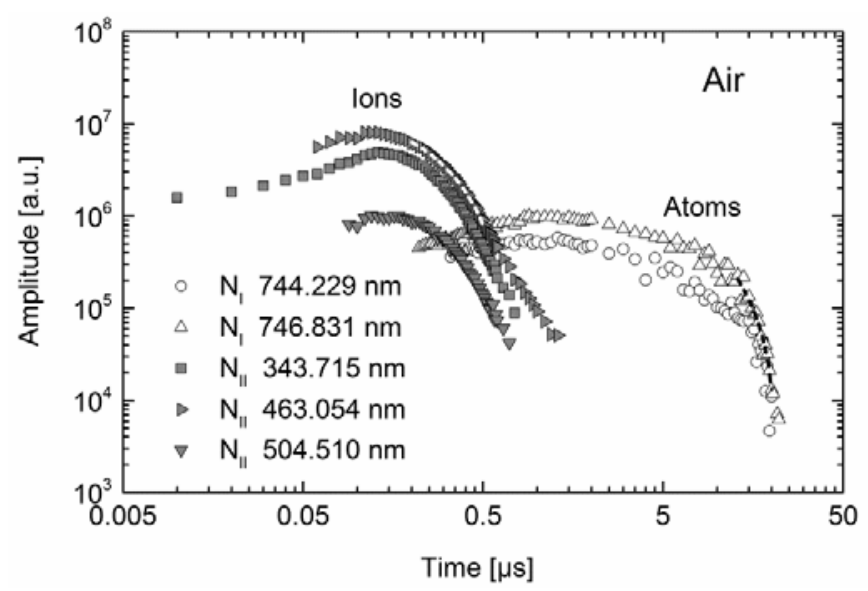

Fig. 13 Exemplary transient line intensities in laser-induced air breakdowns.

a)

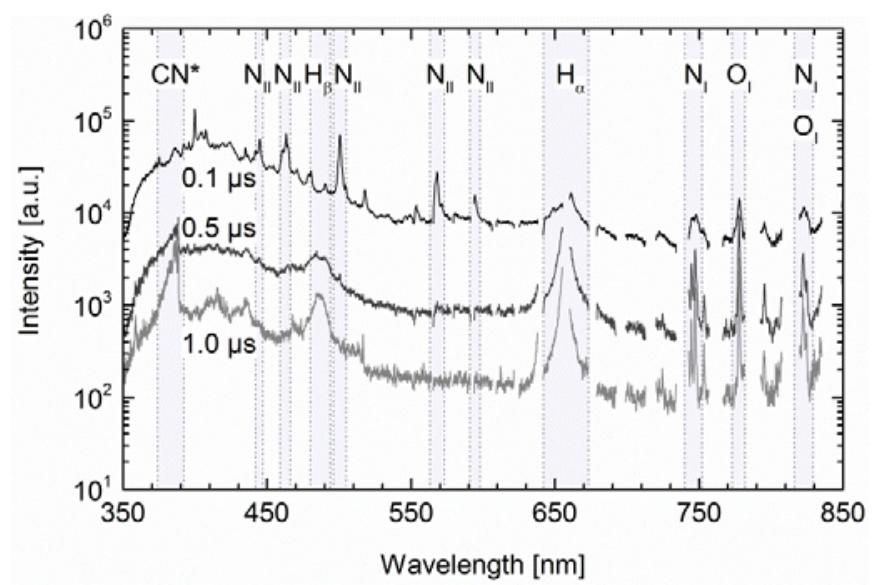


b)

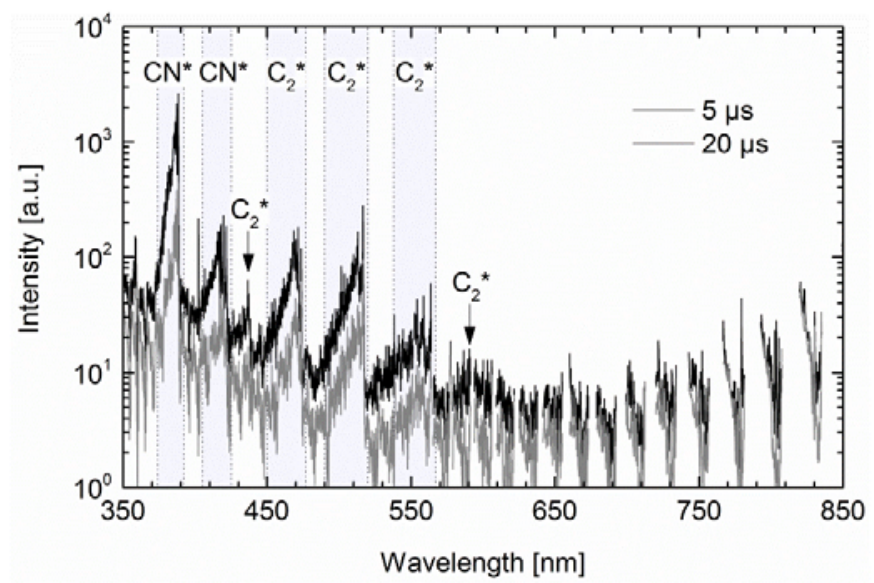

Fig. 14 Optical emission spectra from laser-induced kerosene spray ignitions. Every spectrum is an overlay of twenty individual breakdowns. The exposure time for each spectrum is $100 \mathrm{~ns}$.

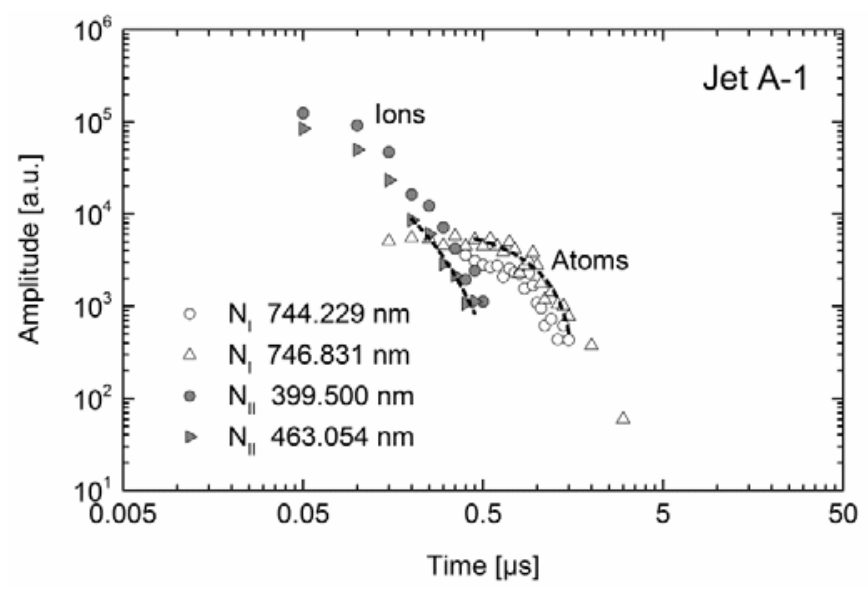

Fig. 15 Exemplary transient line intensities in laser-induced kerosene spray ignitions. 


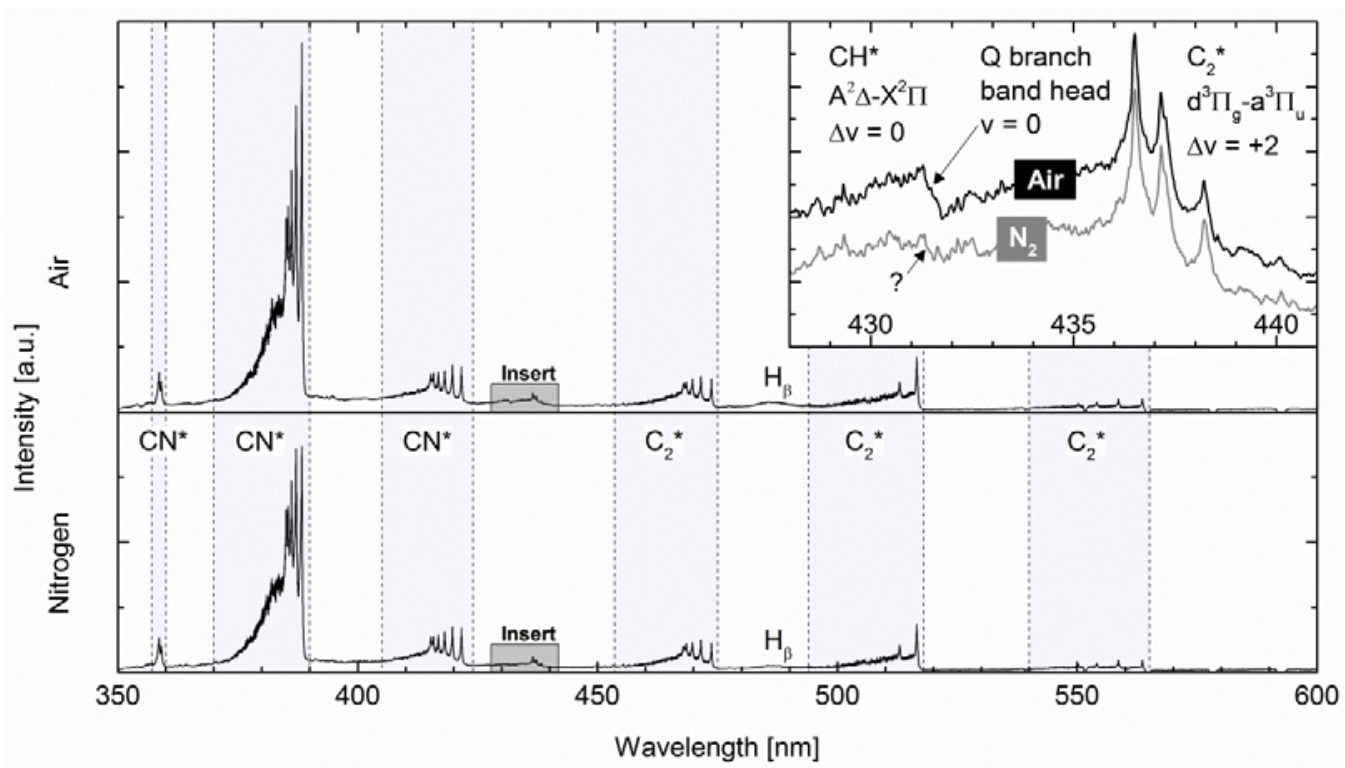

Fig. 16 Comparison of kerosene spray ignitions in air and breakdowns in nitrogen. The exposure time is $100 \mu \mathrm{s}$. The exposures started $1 \mu \mathrm{s}$ after the laser pulse. Every spectrum is an overlay of ten individual breakdowns.

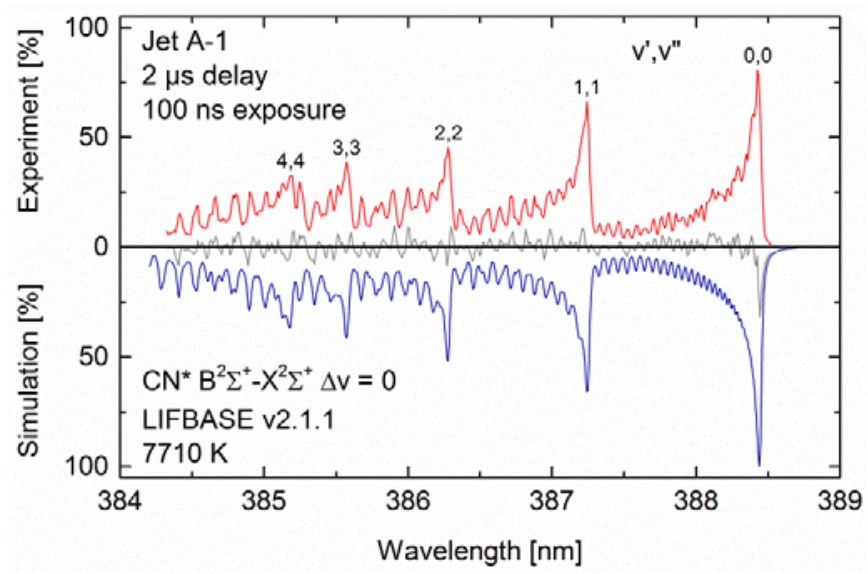

Fig. 17 Comparison of a measured $\mathrm{CN}^{*}$ band sequence and a fitted LIFBASE simulation. 


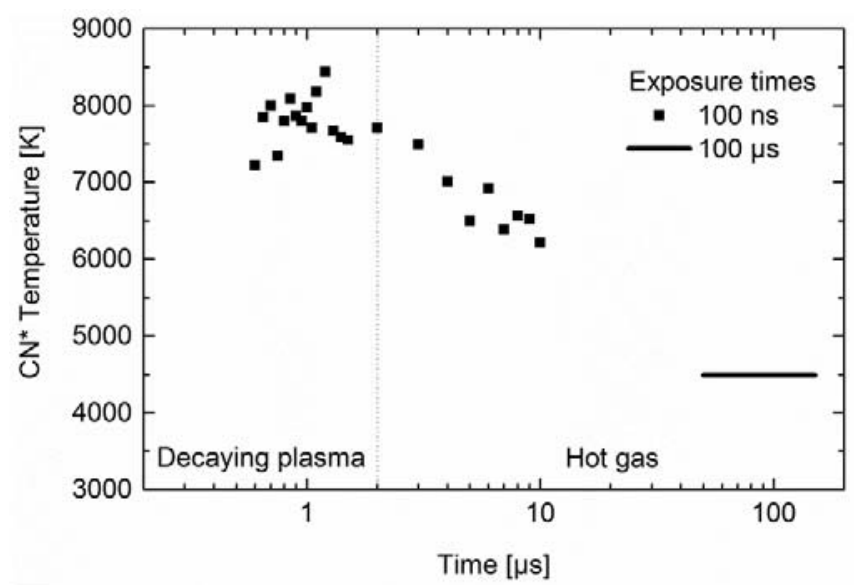

Fig. 18 Estimated rovibrational $\mathrm{CN}^{*}$ temperatures in laser-induced kerosene spray breakdowns during the transition from breakdown plasma to a flame kernel in kerosene spray. 